\title{
Coherent network analysis technique for discriminating gravitational-wave bursts from instrumental noise
}

\author{
Shourov Chatterji, Albert Lazzarini, Leo Stein, and Patrick J. Sutton \\ LIGO-California Institute of Technology, Pasadena, California 91125, USA \\ Antony Searle \\ Australian National University, Canberra, ACT 0200, Australia
}

Massimo Tinto

Jet Propulsion Laboratory, California Institute of Technology, Pasadena, California 91109, USA

(Received 1 May 2006; published 12 October 2006)

\begin{abstract}
The sensitivity of current searches for gravitational-wave bursts is limited by non-Gaussian, nonstationary noise transients which are common in real detectors. Existing techniques for detecting gravitational-wave bursts assume the output of the detector network to be the sum of a stationary Gaussian noise process and a gravitational-wave signal. These techniques often fail in the presence of noise nonstationarities by incorrectly identifying such transients as possible gravitational-wave bursts. Furthermore, consistency tests currently used to try to eliminate these noise transients are not applicable to general networks of detectors with different orientations and noise spectra. In order to address this problem we introduce a fully coherent consistency test that is robust against noise nonstationarities and allows one to distinguish between gravitational-wave bursts and noise transients in general detector networks. This technique does not require any a priori knowledge of the putative burst waveform.
\end{abstract}

DOI: 10.1103/PhysRevD.74.082005

PACS numbers: 04.80.Nn, 07.05.Kf, 95.55.Ym

\section{INTRODUCTION}

Gravitational-wave bursts (GWBs) are among the most exciting classes of signals that large-scale, broadband interferometric gravitational-wave observatories [1-4] will attempt to detect. Potential sources of GWBs include merging compact objects [5-13], core-collapse supernovae [14-18], and gamma-ray burst engines [19]; see [20] for an overview.

A common feature of many GWB-emitting systems is that the signals they produce have not yet been accurately modeled. These signals often depend on complicated (and interesting) physics, such as dynamical gravity and the equation of state of matter at nuclear densities. While this makes GWBs an especially attractive target for study, our lack of knowledge also limits the sensitivity of searches for GWBs. In particular, real detectors exhibit nonGaussian, nonstationary noise "glitches." Current GWB detection schemes assume the detector output to consist of stationary Gaussian background noise plus (possibly) a transient GWB; noise glitches which sometimes occur in coincidence in multiple detectors are found by these detection schemes and falsely appear as candidate GWBs. In matched-filter searches, where the signal waveform is assumed to be known a priori, the $\chi^{2}$ of the fit of the predicted waveform to the data can be used to eliminate noise glitches; see for example [21,22]. For GWBs, however, current source models are not sufficiently accurate to permit such a comparison. Without a robust means of distinguishing GWBs from noise glitches, reducing the false alarm rate requires raising the search detection thresholds, thereby limiting the sensitivity to real, weak GWBs. Consistency tests that can distinguish between GWBs and noise glitches would allow us to avoid this sensitivity loss. Furthermore, the ability to eliminate terrestrial noise and establish the astrophysical origin of a signal will be valuable for making a confident first detection of gravitational waves.

To combat noise glitches, recent GWB searches by the LIGO Scientific Collaboration [23,24] have introduced consistency tests that are designed to accept true GWBs while rejecting noise glitches. The first is a cross correlation test based on Pearson's $r$ statistic [25]. For each a candidate signal, the data from the detectors are whitened, and the correlations between each detector pair are computed. The candidate signal is considered a possible GWB if a certain measure of the combined signficance of the correlations exceeds a threshold. Since the $r$-statistic test is insensitive to the overall amplitude of the transients in the different detectors, a second test then compares the signal amplitude as measured by the two co-aligned LIGOHanford detectors (which should see the same amplitude for a GWB).

These consistency tests have been shown to be effective at reducing the false alarm rate in the LIGO detector network with little loss in detection efficiency. However, they also have significant weaknesses that limit their applicability to more general networks. For example, thresholding on cross correlations effectively assumes that any GWB signal has only a single independent polarization. Furthermore, the $r$-statistic test does not take into account differences in the antenna response of detectors in the 
network, or differences in the detector noise spectra. Similarly, the amplitude consistency test is only effective for the co-aligned detectors at Hanford, so that amplitude information from the Livingston detector is not used. In essence, these tests are based on the assumption that one can ignore differences in orientation (antenna response) and noise levels between detectors in a network. As a consequence, these tests have not been applied in GWB searches with more general networks, such as the LIGOTAMA network [26], and are fundamentally unable to make the most effective use of the new global network of gravitational-wave observatories.

In this article we introduce a new consistency test for discriminating GWBs from noise glitches in networks of gravitational-wave detectors. This test is fully coherent in that it combines data (using both amplitude and phase) from multiple detectors, taking into account the time delays and antenna responses appropriate for the source sky position, the noise spectra of the detectors, and the twopolarization nature of GWBs. It is based on the principles of coherent network analysis for GWBs and takes full advantage of the global detector network.

In order to gain insight into our consistency test, it is useful to review briefly some basic concepts behind coherent network analysis for GWBs. Coherent analysis of data from a network of detectors spaced across the globe inherently provides much more information about the gravitational wave and its source than can be obtained from colocated and co-aligned detectors. With a network of three or more interferometers, for instance, one has in principle sufficient information for good signal-to-noise ratios (SNRs) to infer the direction to the source. This information could be crucial for corroborating a detection with electromagnetic observations (optical, $x$ ray, or radio) and extracting the maximum scientific information from a gravitational-wave signal. Such a network also has enough information to reconstruct the two independent polarization amplitudes of the wave. This is possible because three interferometers provide three independent measures of the gravitational wave (which are functions of time) and two independent time delays. If we consider the geometrical plane passing through the three sites of the interferometers, the two independent time delays jointly identify two possible points in the sky where the signal could have come from that are mirror images of each other with respect to this plane; see Fig. 1. Since the detector antenna patterns are not symmetric with respect to this plane, it is further possible to resolve this two-fold ambiguity by properly accounting for the antenna pattern asymmetry in the analysis of the data. Once the sky position has been determined it is straightforward to make a minimum variance estimate of the two-polarization waveform of the GWB as linear combinations of the detector data streams. The extraction of this information from the combined responses of the individual detectors of the network is called the "solution of the inverse problem" in gravitational-wave astronomy.

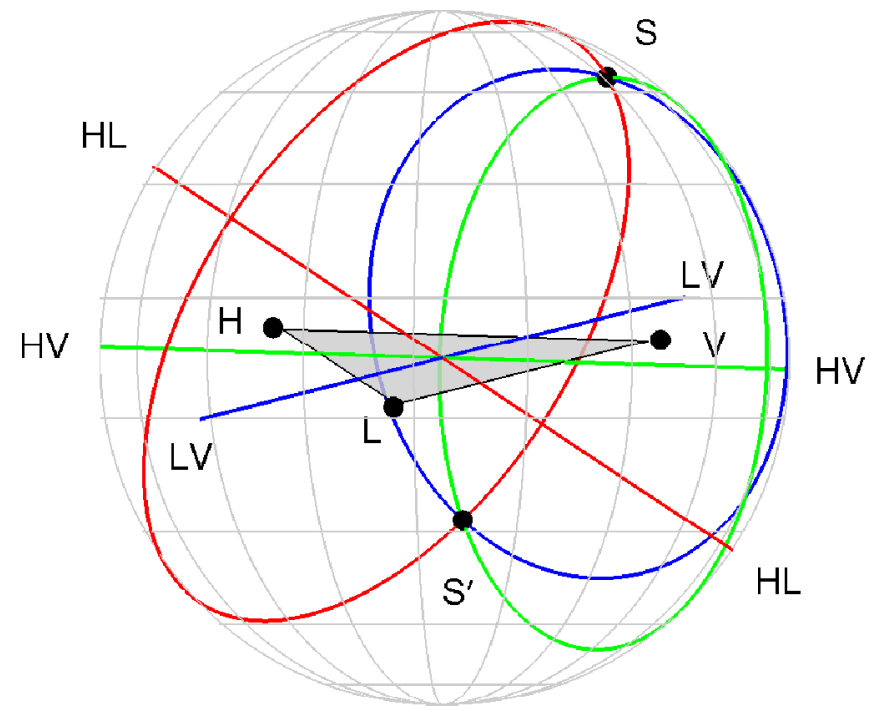

FIG. 1 (color online). Geometry of the network and travel times spent by a GWB to propagate across a three-detector network (detectors H, L, and V). The locus of constant time delay between two detectors form a ring on the sky concentric about the baseline between the two sites. For three detectors, these rings may intersect in two locations. One is the true source direction, $S$, while the other $\left(S^{\prime}\right)$ is its mirror image with respect to the geometrical plane passing through the three sites. This two-fold ambiguity can be resolved by further considering the amplitudes of the responses. For four or more detectors there is a unique intersection point of all of the rings.

The inverse problem for gravitational-wave bursts with a network of three wide-band, widely separated detectors was first solved by Gürsel and Tinto [27]. Their technique, which is referred to in recent literature as the null-stream method, relies on the observation that a gravitational-wave burst present in the data of a network of three wide-band detectors must satisfy a unique closure condition. Gürsel and Tinto studied a two-parameter family of linear combinations of the three data sets, in which the two parameters correspond to the two angular coordinates of the hypothesized sky location of the source. They showed that when the two parameters coincide with the true location of the source then the gravitational-wave burst is cancelled precisely in the linear combination. This point is located by applying a least-squares minimization (i.e., a $\chi^{2}$ test) to the linear combination. In [27] it was also shown that this condition holds regardless of the time dependence of the two-polarization waveform of the burst. This remarkable result makes this method very powerful for solving the inverse problem since it does not require a priori knowledge of the burst waveforms.

Flanagan and Hughes [6] and later Anderson, Brady, Creighton, and Flanagan [28] generalized the Gürsel-Tinto coherent analysis using a maximum-likelihood formulation and showed how coherent techniques could be used for signal detection as well as the inverse problem. Several extensions of the maximum-likelihood approach for GWB 
detection have been proposed recently by Klimenko, Mohanty, Rakhmanov, and Mitselmakher [29-32]. Other coherent detection algorithms have been proposed by Sylvestre [33] and by Arnaud et al. [34]; these methods form a coherent sum of the data from different detectors weighted so as to maximize signal energy.

As pointed out in [35-37], the null-stream method could also be used for discriminating gravitational-wave bursts from noise-triggered fluctuations affecting the data of the detectors. Put simply, uncorrelated noise glitches should not cancel in the null stream. In principle, therefore, they can be vetoed by setting a threshold on the maximum allowable $\chi^{2}$ value. In practice, however, a $\chi^{2}$ veto test is vulnerable to effects that prevent precise cancellation of strong GWBs, such as calibration errors. It also can be ineffective for weak glitches, for which there are often sky positions which yield $\chi^{2} \sim 1$ per degree of freedom even though the glitches are uncorrelated. A $\chi^{2}$ threshold which is high enough to pass a GWB with poor data calibration may also pass a weak glitch. A $\chi^{2}$ threshold which is low enough to reject the glitch may also reject the GWB.

In this paper we propose a modified null-stream-based technique for discriminating GWBs from noise glitches. This test compares the energy in the null stream to that expected if the transients in the detectors are uncorrelated. This second energy measure, which we call the incoherent energy, provides an effective measure of the significance of the $\chi^{2}$ test and renders it robust against both calibration uncertainties and weak glitches. Like the Gürsel-Tinto analysis for determining the source direction, our nullstream consistency test does not require any a priori knowledge of the GWB (or glitch) waveforms. Furthermore, while this test is based on the principles of coherent network analysis, it can be applied to candidate GWBs detected by any scheme, whether coherent or incoherent.

Our paper is organized as follows. In Sec. II we show that there exists a very general and elegant procedure for deriving the null stream for an arbitrary number of detectors with colored noise. We then address the issue that the null-stream $\chi^{2}$ alone can not reliably distinguish between a gravitational-wave burst and noise-generated glitches. This is done by introducing a complementary energy measure, the incoherent energy, and demonstrating that GWBs and glitches separate in the two-dimensional space of null and incoherent energies. This allows us to identify and hence veto noise-generated events.

In Sec. III we discuss the results of the numerical simulation of our statistical test applied to the LIGOVirgo 3-detector network. We assume the three interferometers to be working at the LIGO design sensitivity and quantify the ability of our method to distinguish true GWBs from coincident noise glitches. Although our numerical implementation is not optimized for the signals under consideration, it indicates that gravitational-wave bursts observed in each detector with SNRs of about 1020 can reliably be distinguished from noise glitches of similar energy, and that a significant improvement over the statistics based on the null-stream $\chi^{2}$ alone is achieved. Our conclusions and future work plans are presented in Sec. IV.

\section{ANALYSIS}

Three or more detectors provide redundant measurements of the two polarization components $h_{+}, h_{\times}$of a gravitational wave. It is therefore possible to construct linear combinations of the data streams that do not contain any gravitational-wave component, i.e., that consist only of detector noise. In this section we derive these linear combinations, known as "null streams," for networks containing an arbitrary number of detectors whose noises are different and colored. This derivation is a generalization of the method used by Gürsel and Tinto [27] to solve the inverse problem. It is equivalent to the maximumlikelihood formulation of Flanagan and Hughes [6].

\section{A. Conventions}

The conventions used for the notation in this report are described in Table I.

For a plane [38] gravitational-wave incident from a direction $\hat{\Omega}_{s}$, the strain $h_{+, \times}$at the position $\vec{r}_{\alpha}$ is related to that at some (arbitrary) reference position $\vec{r}_{0}$ by

$$
h_{+, \times}\left(t+\Delta t_{\alpha}\left(\hat{\Omega}_{s}\right), \vec{r}_{\alpha}\right)=h_{+, \times}\left(t, \vec{r}_{0}\right),
$$

where the time-delay $\Delta t_{\alpha}\left(\hat{\Omega}_{s}\right)$ is given by

$$
\Delta t_{\alpha}\left(\hat{\Omega}_{s}\right) \equiv \frac{1}{c}\left(\vec{r}_{0}-\vec{r}_{\alpha}\right) \cdot \hat{\Omega}_{s} .
$$

We can therefore compare the gravitational-wave signals measured by detectors at different locations by shifting the time-series data from each detector according to (2), provided we know the sky location of the source.

The time-series signal produced in detector $\alpha$ at $\vec{r}_{\alpha}$ is

$$
\begin{aligned}
d_{\alpha}\left(t+\Delta t_{\alpha}\left(\hat{\Omega}_{s}\right)\right)= & F_{\alpha}^{+}\left(\hat{\Omega}_{s}\right) h_{+}(t)+F_{\alpha}^{\times}\left(\hat{\Omega}_{s}\right) h_{\times}(t) \\
& +n_{\alpha}\left(t+\Delta t_{\alpha}\left(\hat{\Omega}_{s}\right)\right) .
\end{aligned}
$$

Here $n_{\alpha}$ is the stationary strain-equivalent background noise of detector $\alpha$, and $F_{\alpha}^{+}, F_{\alpha}^{\times}$are the antenna response functions [27] for detector $\alpha$ for the sky position $\hat{\Omega}_{s}$ of the gravitational-wave source. For brevity, we write $h_{+, \times}(t) \equiv$ $h_{+, \times}\left(t, \vec{r}_{0}\right)$.

\section{B. Null-stream construction}

Let us assume for the moment that we know the direction $\hat{\Omega}_{s}$ to the source. Then we can time-shift the data from each detector as in (3), and drop explicit references to the time delays $\Delta t_{\alpha}$ and the sky position. Transforming to the 
TABLE I. Notation conventions for commonly used quantities in this paper.

$D$

$\alpha, \beta \in[1, \ldots, D]$

$X$

$\boldsymbol{X}^{T}$

$N$

$j, k \in[0, \ldots, N-1]$

$h_{+}, h_{\times}$

$\hat{\Omega}_{s}$

$\hat{\Omega}$

$\boldsymbol{d}, \boldsymbol{n}, \boldsymbol{F}$

$\boldsymbol{d}_{\mathrm{w}}, \boldsymbol{n}_{\mathrm{w}}, \boldsymbol{F}_{\mathrm{w}}$
Number of detectors in the network Index specifying detector

(Boldface) a vector or matrix on the space of detectors

Matrix transpose of $\boldsymbol{X}$

Number of data samples from each detector being analyzed

Index specifying time or frequency sample

"Plus" and "cross" polarization waveforms of the gravitational wave

Sky position of the gravitational-wave source

Trial sky position

Data, noise, and antenna responses in strain

Noise-weighted (whitened) data, noise, and antenna responses
Fourier domain, Eq. (3) becomes

$$
\tilde{d}_{\alpha}(f)=F_{\alpha}^{+} \tilde{h}_{+}(f)+F_{\alpha}^{\times} \tilde{h}_{\times}(f)+\tilde{n}_{\alpha}(f) .
$$

The Fourier transform and its inverse are defined by

$$
\begin{aligned}
\tilde{d}_{\alpha}(f) & =\int_{-\infty}^{+\infty} d_{\alpha}(t) e^{-2 \pi i f t} d t, \\
d_{\alpha}(t) & =\int_{-\infty}^{+\infty} \tilde{d}_{\alpha}(f) e^{2 \pi i f t} d f .
\end{aligned}
$$

The one-sided strain noise power spectral density $S_{\alpha}(f)$ of the stationary noise $n_{\alpha}$ is given by

$$
\left\langle\tilde{n}_{\alpha}(f) \tilde{n}_{\beta}^{*}\left(f^{\prime}\right)\right\rangle=\frac{1}{2} \delta_{\alpha \beta} \delta\left(f-f^{\prime}\right) S_{\alpha}(f) .
$$

For present detectors, $S_{\alpha}(f)$ is a strongly varying function of frequency. Since it will prove convenient to work with white-noise data, without loss of generality we divide the strain data at each frequency by the estimated amplitude spectrum $\sqrt{S_{\alpha}(f) / 2}$ of the corresponding detector noise. The whitened data $\tilde{d}_{w \alpha}$ is then given by

$$
\begin{aligned}
\tilde{d}_{w \alpha}(f) & \equiv \frac{\tilde{d}_{\alpha}(f)}{\sqrt{S_{\alpha}(\bar{f}) / 2}} \\
& =F_{w \alpha}^{+} \tilde{h}_{+}(f)+F_{w \alpha}^{\times} \tilde{h}_{\times}(f)+\tilde{n}_{w \alpha}(f),
\end{aligned}
$$

where the $n_{w \alpha}(t)$ are unit Gaussian noise processes and $F_{w \alpha}^{+, \times}$are the noise-weighted antenna responses

$$
F_{w \alpha}^{+, \times}\left(\hat{\Omega}_{s}, f\right) \equiv \frac{F_{\alpha}^{+, \times}\left(\hat{\Omega}_{s}\right)}{\sqrt{S_{\alpha}(f) / 2}} .
$$

The $F_{w \alpha}^{+, \times}$contain all of the information on the detector sensitivities, both as functions of frequency and source sky position.

For a network of $D$ detectors, Eq. (7) can be written in the equivalent matrix form

$$
\left[\begin{array}{c}
\tilde{d}_{w 1} \\
\tilde{d}_{w 2} \\
\vdots \\
\tilde{d}_{w D}
\end{array}\right]=\left[\begin{array}{cc}
F_{w 1}^{+} & F_{w 1}^{\times} \\
F_{w 2}^{+} & F_{w 2}^{\times} \\
\vdots & \vdots \\
F_{w D}^{+} & F_{w D}^{\times}
\end{array}\right]\left[\begin{array}{c}
\tilde{h}_{+} \\
\tilde{h}_{\times}
\end{array}\right]+\left[\begin{array}{c}
\tilde{n}_{w 1} \\
\tilde{n}_{w 2} \\
\vdots \\
\tilde{n}_{w D}
\end{array}\right],
$$

or

$$
\tilde{\boldsymbol{d}}_{\mathrm{w}}=\boldsymbol{F}_{\mathrm{w}} \tilde{\boldsymbol{h}}+\tilde{\boldsymbol{n}}_{\mathrm{w}},
$$

where we use boldface to denote vectors and matrices. Here

$$
\tilde{\boldsymbol{h}} \equiv\left[\begin{array}{c}
\tilde{h}_{+} \\
\tilde{h}_{\times}
\end{array}\right],
$$

and the matrix $\boldsymbol{F}_{\mathrm{w}}$ is defined as

$$
\boldsymbol{F}_{\mathrm{w}}\left(\hat{\Omega}_{s}, f\right) \equiv\left[\begin{array}{ll}
\boldsymbol{F}_{\mathrm{w}}^{+} & \boldsymbol{F}_{\mathrm{w}}^{\times}
\end{array}\right]=\left[\begin{array}{cc}
F_{\mathrm{w} 1}^{+} & F_{\mathrm{w} 1}^{\times} \\
F_{\mathrm{w} 2}^{+} & F_{\mathrm{w} 2}^{\times} \\
\vdots & \vdots \\
F_{\mathrm{w} D}^{+} & F_{\mathrm{w} D}^{\times}
\end{array}\right] .
$$

This form makes it clear that, regardless of the functional form of $\tilde{h}_{+, \times}$, the gravitational-wave burst can only contribute to the network output along the directions $\boldsymbol{F}_{\mathrm{w}}^{+}$and $\boldsymbol{F}_{\mathrm{w}}^{\times}$. The construction of null streams is thus obvious: we simply project the data $\tilde{\boldsymbol{d}}$ orthogonally to these directions. Formally, we select a new orthonormal Cartesian coordinate basis $\boldsymbol{e}_{i}$ for the space of $\tilde{\boldsymbol{d}}$ in which vectors $\boldsymbol{e}_{D-1}$ and $\boldsymbol{e}_{D}$ span $\boldsymbol{F}_{\mathrm{w}}^{+}$and $\boldsymbol{F}_{\mathrm{w}}^{\times}$. The remaining basis vectors $\boldsymbol{e}_{1}, \ldots, \boldsymbol{e}_{D-2}$ are then orthogonal to $\boldsymbol{F}_{\mathrm{w}}^{+}$and $\boldsymbol{F}_{\mathrm{w}}^{\times}$,

$$
\boldsymbol{F}_{\mathrm{w}}^{+} \cdot \boldsymbol{e}_{i}=0=\boldsymbol{F}_{\mathrm{w}}^{\times} \cdot \boldsymbol{e}_{i} \quad i \in\{1, \ldots, D-2\} .
$$

We say that the $\boldsymbol{e}_{i=1, \ldots, D-2}$ form an orthonormal basis for the null space of $\boldsymbol{F}_{\mathrm{w}}{ }^{T}$ [39], hence the term "null-stream formalism." We then construct a $(D-2) \times D$ matrix $\boldsymbol{A}$ whose rows are the components of this orthonormal basis,

$$
\boldsymbol{A}\left(\hat{\Omega}_{s}, f\right) \equiv\left[\begin{array}{c}
\boldsymbol{e}_{1}^{T} \\
\vdots \\
\boldsymbol{e}_{D-2}^{T}
\end{array}\right] .
$$


By construction $\boldsymbol{A}$ is orthogonal to $\boldsymbol{F}_{\mathrm{w}}^{+}$and $\boldsymbol{F}_{\mathrm{w}}^{\times}$, so

$$
\boldsymbol{A} \boldsymbol{F}_{\mathrm{w}}=0 .
$$

We obtain the null streams $\tilde{z}$ by applying $\boldsymbol{A}$ to the network data vector:

$$
\tilde{\boldsymbol{z}} \equiv \boldsymbol{A} \tilde{\boldsymbol{d}}_{\mathrm{w}}=\boldsymbol{A} \boldsymbol{F}_{\mathrm{w}} \tilde{\boldsymbol{h}}+\boldsymbol{A} \tilde{\boldsymbol{n}}_{\mathrm{w}}=\boldsymbol{A} \tilde{\boldsymbol{n}}_{\mathrm{w}} .
$$

The two independent strain components $\tilde{h}_{+, \times}$are canceled out as a consequence of the definition (13) of the null space, making each $\tilde{z}_{\alpha}$ a null stream.

For example, the three-detector case considered by Gürsel and Tinto [27] is particularly simple. In this case the matrix $\boldsymbol{A}$ is equal to

$$
\boldsymbol{A}=\frac{\boldsymbol{F}_{\mathrm{w}}^{+} \times \boldsymbol{F}_{\mathrm{w}}^{\times}}{\left|\boldsymbol{F}_{\mathrm{w}}^{+} \times \boldsymbol{F}_{\mathrm{w}}^{\times}\right|} .
$$

This is illustrated schematically in Fig. 2. In the general case $\boldsymbol{A}$ can be obtained via singular value decomposition [40] or by explicitly constructing the associated projection operator (see [32] or Appendix B for details).

Note that $\boldsymbol{A}$ is a function of the sky position [through $F_{\alpha}^{+, \times}\left(\hat{\Omega}_{s}\right)$ ] and frequency [through $\left.S_{\alpha}(f)\right]$. The GWB will only be cancelled when $\boldsymbol{A}$ is evaluated for the correct source location, since generally

$$
\boldsymbol{A}(\hat{\Omega}, f) \boldsymbol{F}_{\mathrm{w}}\left(\hat{\Omega}^{\prime}, f\right) \neq 0 .
$$

In the next subsection we will discuss how to deal with the case where the source location is not known.

In the preceding discussion we have assumed implicitly that $\boldsymbol{F}_{\mathrm{w}}^{+}$and $\boldsymbol{F}_{\mathrm{w}}^{\times}$are independent. In the general case the number of independent null streams is $D-r$, where $r$ is the number of independent columns of $\boldsymbol{F}$, i.e.

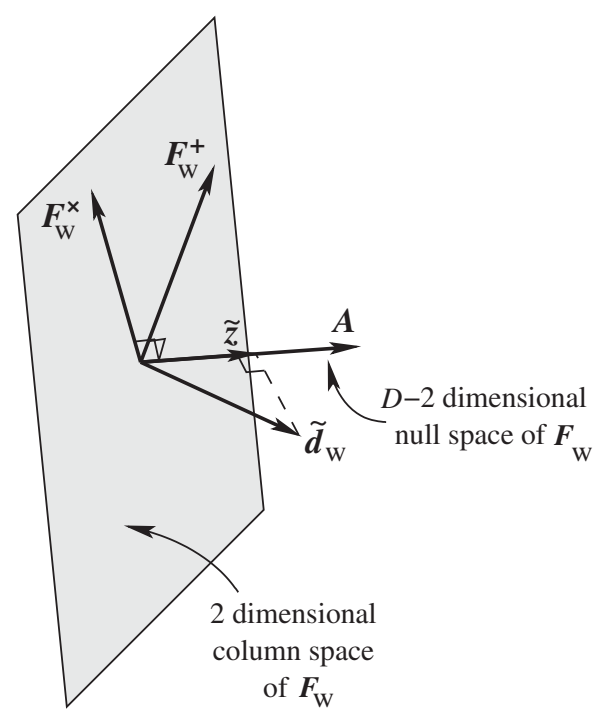

FIG. 2. Geometry of the null-stream construction for the 3detector case. The null stream is obtained by projecting the data along the vector $\boldsymbol{A}$, which is orthogonal to $\boldsymbol{F}_{\mathrm{w}}^{+}$and $\boldsymbol{F}_{\mathrm{w}}^{\times}$. For $D$ nonaligned detectors $\boldsymbol{A}$ has $D-2$ dimensions.

$$
r \equiv \operatorname{rank}\left(\boldsymbol{F}_{\mathrm{w}}\right) .
$$

There are two cases:

(1) If at least one detector in the network has a different alignment from the others then $\boldsymbol{F}_{\mathrm{w}}^{+}$and $\boldsymbol{F}_{\mathrm{w}}^{\times}$are independent and $\operatorname{rank}\left(\boldsymbol{F}_{\mathrm{w}}\right)=2$. In this case there are $D-2$ null streams, and the method is applicable to networks of $D \geq 3$ detectors.

(2) If all detectors in the network are aligned then $\boldsymbol{F}_{\mathrm{w}}^{+} \propto$ $\boldsymbol{F}_{\mathrm{w}}^{\times}$and $\operatorname{rank}\left(\boldsymbol{F}_{\mathrm{w}}\right)=1$. In this case there are $D-1$ null streams, and the method is applicable to networks of $D \geq 2$ detectors. Although in this paper we will concentrate on case 1, i.e. three or more nonaligned interferometers, we emphasize that our method works also with aligned detectors.

\section{Null-stream analysis}

Since the $\boldsymbol{e}_{i}$ are orthonormal by construction, it follows that

$$
\boldsymbol{A} \boldsymbol{A}^{T}=\boldsymbol{I}_{(D-2) \times(D-2)} .
$$

This implies that for a GWB each null-stream $\tilde{z}_{\alpha}$ is a Gaussian random process of unit variance and is uncorrelated with $\tilde{z}_{\beta}$, for all $\beta \neq \alpha$. This is the main advantage of the null-stream formalism over incoherent techniques: the noise distribution of the projected network data is known a priori, regardless of the form of, $h_{+, \times}$, under the assumption that a GWB from a particular direction is present. This allows us to perform statistically significant tests of the network data, in particular, to test the hypothesis that a GWB from a given direction is present.

Since data from gravitational-wave detectors are sampled and digitized, in what follows we will consistently use discrete notation in our analysis of the statistics of the null stream. Our conventions for discretely sampled data are as follows: the Fourier transform pair becomes

$$
\tilde{x}[k]=\sum_{j=0}^{N-1} x[j] \mathrm{e}^{-i 2 \pi j k / N}, \quad x[j]=\frac{1}{N} \sum_{k=0}^{N-1} \tilde{x}[k] \mathrm{e}^{i 2 \pi j k / N},
$$

where $N$ is the number of data points in the time domain. Denoting the sampling rate by $f_{s}$, we can convert from continuous to discrete notation using $x(t) \rightarrow x[j], \tilde{x}(f) \rightarrow$ $f_{s}^{-1} \tilde{x}[k], \int d t \rightarrow f_{s}^{-1} \sum_{j}, \int d f \rightarrow f_{s} N^{-1} \sum_{k}, \quad \delta\left(t-t^{\prime}\right) \rightarrow$ $f_{s} \delta_{j j^{\prime}}$, and $\delta\left(f-f^{\prime}\right) \rightarrow N f_{s}^{-1} \delta_{k k^{\prime}}$. For example, the onesided strain noise power spectrum $S_{\alpha}[k]$ is

$$
\left\langle\tilde{n}_{\alpha}^{*}[k] \tilde{n}_{\beta}\left[k^{\prime}\right]\right\rangle=\frac{N}{2} \delta_{\alpha \beta} \delta_{k k^{\prime}} S_{\alpha}[k] .
$$

We will whiten the data by applying a zero-phase whitening filter [41,42], and our normalization convention for whitened data is

$$
\left\langle\tilde{n}_{\mathrm{w} \alpha}^{*}[k] \tilde{n}_{\mathrm{w} \beta}\left[k^{\prime}\right]\right\rangle=\delta_{\alpha \beta} \delta_{k k^{\prime}} .
$$


The energy in the null streams is

$$
E_{\text {null }} \equiv \sum_{\alpha=1}^{D-r} \sum_{k=0}^{N-1}\left|\tilde{z}_{\alpha}[k]\right|^{2} .
$$

Using (20) and (23) it follows that at the true source position $2 E_{\text {null }}$ is $\chi^{2}$ distributed with $2 N(D-r)$ degrees of freedom. In this case the expectation value of the null energy and its variance are both $N(D-r)$.

Although our considerations so far have assumed the sky position $\hat{\Omega}_{s}$ of the GWB source to be known a priori, in practice this may not be the case. Since we know that at the correct source location the null energy will not contain any contribution from the signal, a straightforward procedure is to scan over a grid of sky positions in search of the minimum of the null energy. Gürsel and Tinto [27] used time-delay estimates to limit their search to two possible regions of the sky (the regions around points $S$ and $S^{\prime}$ in Fig. 1). This approach is not applicable when the timing uncertainty is of the same order as the light travel time along the shortest baseline between detectors in the network, thus necessitating an all-sky search. (For example, for the LIGO-Virgo network we consider, the shortest baseline is $10 \mathrm{~ms}$. The timing uncertainty for each detector in a recent GWB search [23] was $[-2,5]$ ms systematic plus $3 \mathrm{~ms}$ statistical. An all-sky scan would be required for coherent testing of events from this search.) Since the numerical analysis in [27] implies that the characteristic angular width of the minimum of the null energy in a neighborhood of the source location for a GWB with a central frequency of about $100 \mathrm{~Hz}$ is equal to approximately $10^{-2}$ steradian for high SNRs, it follows that an allsky search should be performed over a sky grid containing at least $4 \pi / 10^{-2}$ resolvable directions. (Our numerical tests use a conservatively oversampling grid of $10^{4}$ points.) In either case, for each trial direction one postulates the presence of a gravitational-wave signal, forms a linear combination of the detectors that is orthogonal to a GWB from that postulated direction, and $\chi^{2}$-tests this null stream for excess energy. If there exists a particular direction for which there is no excess energy in the null stream, the data is regarded as consistent with the hypothesis that the transient is a gravitational-wave burst incoming from the inferred direction. If, on the other hand, $E_{\text {null }}$ is inconsistent with a $\chi^{2}$ distribution, then one rejects the hypothesis that the transient is a GWB incoming from that direction. The best estimate of the source direction is taken as the direction with minimum $\chi^{2}$.

Although the null-stream method does not require knowledge of the two GWB waveforms for its implementation, once the source location $\hat{\Omega}_{s}$ has been identified it is straightforward to reconstruct $h_{+, \times}$from the data themselves [27] (if the detectors are all aligned then only one of the polarizations can be reconstructed.) For completeness, the minimum-variance estimate of the two waveforms for a network containing an arbitrary number of nonaligned detectors is given in Appendix A; see also, for example, [32].

The null-stream combination of the data from a threedetector network and the resulting $\chi^{2}$ test were first derived (in a different way) by Gürsel and Tinto [27] for detectors whose noises are white. They also implemented a nearoptimal filtering procedure to account for colored noise. Our approach builds on this by generalizing and simplifying the derivation of the null-stream and waveform reconstruction to networks containing an arbitrary number of detectors with different colored noises. One can also show that our geometrical null-stream construction procedure is formally equivalent to the maximum-likelihood analysis originally presented in [6]; see [32].

\section{Distinguishing GWBs from noise transients}

The numerical analysis performed by Gürsel and Tinto in [27] was not aimed at checking whether the null energy estimator could distinguish GWBs from noise-induced glitches. In fact, an analysis based purely on the null stream runs into difficulty when applied to data containing noise transients. Strong uncorrelated glitches generally will not cancel in the null-stream combination because they are not correlated in amplitude and phase in a way consistent with a GWB, implying a $\chi^{2}>1$ per degree of freedom. However, a null-stream analysis of a real GWB may also produce $\chi^{2}>1$ per degree of freedom, due to imperfect cancellation of the GWB in the null stream. This may happen for various reasons, such as the use of a discrete sky grid, inaccurate calibration of the data, or imperfect whitening of nonstationary data. Thus, an analysis based purely on the null stream would be forced to either reject both glitches and GWBs or accept both. Further, counter to one's intuition, this problem could get worse with stronger signals.

Glitches can also fool a null-stream analysis in 3detector networks when the transient is weak in at least one detector. This is because the nonobservation of a signal by one detector $\alpha$ is always consistent with a GWB incident from a direction and polarization to which the detector responses $F_{\mathrm{w} \alpha}^{+}$and $F_{\mathrm{w} \alpha}^{\times}$are sufficiently small. For sky positions with $F_{\mathrm{w} \alpha}^{+} \sim F_{\mathrm{w} \alpha}^{\times} \sim 0$, the null-stream projection matrix $\boldsymbol{A}$ reduces to

$$
\boldsymbol{A}_{\beta \gamma} \rightarrow \delta_{\alpha \beta} \delta_{\alpha \gamma}, \quad F_{\mathrm{w} \alpha}^{+}, F_{\mathrm{w} \alpha}^{\times} \rightarrow 0 .
$$

That is, the null stream for this sky position reduces to the detector in which there is no transient. This gives a $\chi^{2}$ per degree of freedom of order unity regardless of whether the transient in the other two detectors is due to a GWB or noise. (Equivalently, when only two detectors observe a signal, it is always possible to find $h_{+, \times}$that fit the output of these two detectors.) Thus double-coincident glitches will always pass a $\chi^{2}$ test for certain areas on the sky. And while networks containing four or more detectors will be less affected by this problem [43] because the size of the 
region of the sky producing two or more simultaneous, below-threshold responses is smaller than that for a single response, it will not be null.

In what follows we propose a simple way to make nullstream analyses robust against glitches by comparing the amount of energy in the null streams to that expected if the transients are uncorrelated. Let us consider how the nullstream energy $E_{\text {null }}$ depends on the individual detector data streams $d_{\mathrm{w} \alpha}$. By defining the matrix

$$
\boldsymbol{Q} \equiv \boldsymbol{A}^{T} \boldsymbol{A},
$$

we may write (24) in the convenient form

$$
\begin{aligned}
& E_{\mathrm{null}}(\hat{\Omega})=\sum_{k=0}^{N-1} \sum_{\alpha=1}^{D} \sum_{\beta=1}^{D} \tilde{d}_{\mathrm{w} \alpha}^{*}[k] Q_{\alpha \beta}[k, \hat{\Omega}] \tilde{d}_{\mathrm{w} \beta}[k] \\
= & \sum_{k=0}^{N-1}\left[\begin{array}{lll}
\tilde{d}_{\mathrm{w} 1}^{*} & \ldots & \tilde{d}_{\mathrm{w} D}^{*}
\end{array}\right]\left[\begin{array}{cccc}
Q_{11} & Q_{12} & \ldots & Q_{1 D} \\
Q_{21} & Q_{22} & & Q_{2 D} \\
\vdots & & & \vdots \\
Q_{D 1} & Q_{D 2} & \ldots & Q_{D D}
\end{array}\right] \\
& \times\left[\begin{array}{c}
\tilde{d}_{\mathrm{w} 1} \\
\tilde{d}_{\mathrm{w} 2} \\
\vdots \\
\tilde{d}_{\mathrm{w} D}
\end{array}\right] .
\end{aligned}
$$

Note that the null energy contains contributions from both cross correlation $\left(\tilde{d}_{\mathrm{w} \alpha}^{*} \tilde{d}_{\mathrm{w} \beta}\right)$ and autocorrelation $\left(\tilde{d}_{\mathrm{w} \alpha}^{*} \tilde{d}_{\mathrm{w} \alpha}\right)$ terms. If the signals in the various detectors are independent (as one might expect for noise glitches), then the expectation value of the cross correlation terms in (28) will be small compared to that of the autocorrelation terms. In this case the expectation value of $E_{\text {null }}$ is just the sum of the diagonal terms in (28):

$$
\text { mean }\left(E_{\mathrm{null}}(\hat{\Omega})\right) \rightarrow \sum_{k=0}^{N-1} \sum_{\alpha=1}^{D} Q_{\alpha \alpha}[k, \hat{\Omega}]\left|\tilde{d}_{\mathrm{w} \alpha}[k]\right|^{2} .
$$

Here the mean is an ensemble average over noise instantiations. This observation motivates the use of a new energy measure, the incoherent energy $E_{\mathrm{inc}}$, defined as the autocorrelation contribution to the null energy:

$$
E_{\mathrm{inc}}(\hat{\Omega}) \equiv \sum_{k=0}^{N-1} \sum_{\alpha=1}^{D} Q_{\alpha \alpha}[k, \hat{\Omega}]\left|\tilde{d}_{\mathrm{w} \alpha}[k]\right|^{2} .
$$

If the transient signals in the various detectors are not correlated, then we expect the following approximate equality to hold:

$$
\operatorname{mean}\left(E_{\text {null }}\right) \simeq \operatorname{mean}\left(E_{\text {inc }}\right) .
$$

If instead the signals in the detectors are due to a GWB, then at the correct sky location the GWB contributions cancel in the null energy, and the following inequality should hold:

$$
\operatorname{mean}\left(E_{\text {null }}\right)<\operatorname{mean}\left(E_{\text {inc }}\right) .
$$

Thus, a distinguishing feature of a GWB is that a significant fraction of the energy in the individual detector data streams is cancelled in the null stream.

In our simulations we test two simple measures of the degree to which $E_{\text {null }}$ and $E_{\text {inc }}$ show the behavior expected of a GWB. These are the quantities $E_{\text {null }}-E_{\text {inc }}$ and $\left(E_{\text {null }}-E_{\text {inc }}\right) / E_{\text {inc }}=E_{\text {null }} / E_{\text {inc }}-1$, which represent, respectively, the amount of "correlated energy" and the ratio of correlated energy to "uncorrelated energy" in the data. For reference, we will also test $E_{\text {null }}$ alone.

An example is shown in Fig. 3. This figure shows the incoherent energy versus null energy for a GWB and a glitch of the same amplitude, evaluated for approximately $10^{4}$ uniformly distributed sky locations (see Sec. III for details). We note that for both the GWB and the glitch there are sky positions for which the null energy is approximately 1 per degree of freedom, so that the null energy alone does not distinguish this glitch and GWB. However, glitches and GWBs scatter differently in terms of the two energy measures $E_{\text {null }}$ and $E_{\text {inc }}$. This suggests a modified procedure for distinguishing GWBs from glitches: scan over the sky and look for directions for which $E_{\text {null }}$ is significantly smaller than $E_{\text {inc }}$. If there exists a sky direction for which $E_{\text {null }}$ is sufficiently small compared to $E_{\text {inc }}$, we conclude that the transient is consistent with a GWB. If instead there is no direction for which $E_{\text {null }}$ is sufficiently small compared to $E_{\text {inc }}$, we conclude that the transient is not a GWB.

Strong GWBs that are not precisely cancelled in the null stream (due to calibration errors, for example) and have $E_{\text {null }}>N(D-r)$ will still pass this test because $E_{\text {null }}<$ $E_{\text {inc }}$. Double-coincident glitches will fail even though $E_{\text {null }} \simeq N(D-r)$ because they have $E_{\text {null }} \simeq E_{\text {inc }}$. Put another way, the incoherent energy provides a natural cutoff in the significance of a $\chi^{2}$ per degree of freedom measurement. Intuitively, if the null stream cancels out some large fraction of the excess incoherent energy, then we expect the event to be a gravitational wave, even if $\chi^{2}>1$ per degree of freedom. The failure to cancel a significant portion of the incoherent energy will eliminate glitches even if $\chi^{2} \simeq 1$ per degree of freedom.

To get more insight into the behavior and usefulness of these energy measures, let us consider in more detail their expectation values over noise instantiations. Allowing for the case in which the transient is not a GWB, we write

$$
\tilde{d}_{\mathrm{w} \alpha}[k]=\tilde{n}_{\mathrm{w} \alpha}[k]+\tilde{g}_{\mathrm{w} \alpha}[k],
$$

where $g_{\mathrm{w} \alpha}$ denotes the noise-weighted transient as seen in detector $\alpha$. For example, if the transient is a GWB from the direction $\hat{\Omega}_{s}$, and the tested sky position is $\hat{\Omega}$, then 

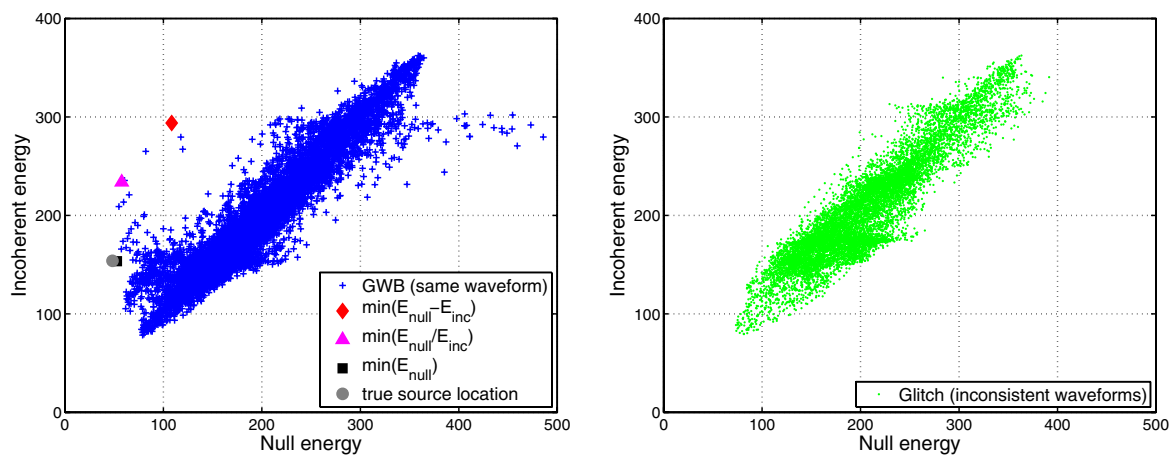

FIG. 3 (color online). Scatter plot of the null and incoherent energies for a simulated GWB (left) and a simulated glitch (right) seen in a network consisting of the LIGO-Hanford and LIGO-Livingston $4 \mathrm{~km}$ detectors and Virgo. In both cases the signal was scaled to rms SNR of 20 over the three detectors. Each point represents one trial sky position; approximately $10^{4}$ sky positions in a uniform grid were tested. The waveforms and detector network used are discussed in detail in Sec. III. The GWB and glitch signals have the same signal-to-noise ratios and time delays in the individual detectors and so are indistinguishable to incoherent tests. Note that for both the GWB and the glitch there are sky positions for which the null energy is consistent with noise $\left[E_{\text {null }} \simeq N(D-r)=60\right.$ for these simulations]. However, for the GWB there are also sky positions with $E_{\text {null }} \ll E_{\text {inc }}$ (points above the diagonal); these are due to the fact that the GWB signal is correlated between the detectors. The glitch signal does not access this portion of the $\left(E_{\text {null }}, E_{\text {inc }}\right)$ space. This observation is the basis of our consistency test; we scan over the sky and look for directions where $E_{\text {null }}<E_{\text {inc }}$. The true source location is indicated by the circle on the GWB plot. Also shown on the GWB plot are the tested sky positions which have the smallest values of $E_{\text {null }}-E_{\mathrm{inc}}, E_{\mathrm{null}} / E_{\mathrm{inc}}$, and $E_{\text {null }}$. The sky maps for these same simulations are shown in Fig. 4.

$$
\begin{aligned}
\tilde{g}_{\mathrm{w} \alpha}[k]= & \frac{F_{\alpha}^{+}\left[k, \hat{\Omega}_{s}\right] \tilde{h}_{+}[k]+F_{\alpha}^{\times}\left[k, \hat{\Omega}_{s}\right] \tilde{h}_{\times}[k]}{\sqrt{\frac{N}{2} S_{\alpha}[\bar{L}]}} \\
& \times \mathrm{e}^{i 2 \pi f_{s} k / N\left(\Delta t_{\alpha}(\hat{\Omega})-\Delta t_{\alpha}\left(\hat{\Omega}_{s}\right)\right)} .
\end{aligned}
$$

The phase term accounts for time-shifting based on the incorrect sky location $\hat{\Omega}$ instead of the true but unknown sky location $\hat{\Omega}_{s}$.

For simplicity, let us restrict ourselves to the case of detectors with equal noise spectra, for which $\boldsymbol{Q}$ is independent of frequency. Then the noise-weighted transient signal $g_{\mathrm{w} \alpha}$ appears in the null and incoherent energies in the combination

$$
\rho_{\alpha \beta}^{2} \equiv \sum_{k=0}^{N-1} \tilde{g}_{\mathrm{w} \alpha}^{*}[k] \tilde{g}_{\mathrm{w} \beta}[k]=\sum_{k=0}^{N-1} \frac{\tilde{g}_{\alpha}^{*}[k] \tilde{g}_{\beta}[k]}{\frac{N}{2} \sqrt{S_{\alpha}[k] S_{\beta}[k]}},
$$

which is the noise-weighted cross correlation of the signals in detectors $\alpha$ and $\beta$. The diagonal terms are

$$
\begin{aligned}
\rho_{\alpha \alpha}^{2} & \equiv \sum_{k=0}^{N-1}\left|\tilde{g}_{\mathrm{w} \alpha}[k]\right|^{2}=\sum_{k=0}^{N-1} \frac{\left|\tilde{g}_{\alpha}[k]\right|^{2}}{\frac{N}{2} S_{\alpha}[k]} \\
& \leftrightarrow 2 \int_{-\infty}^{\infty} d f \frac{\left|\tilde{g}_{\alpha}(f)\right|^{2}}{S_{\alpha}(f)} .
\end{aligned}
$$

This autocorrelation term is simply the squared signal-tonoise ratio of an optimal matched filter for the transient in detector $\alpha$, as indicated by the second line of (36).

As an example, let us consider the special case of a linearly polarized GWB (e.g., with $h_{\times}=0$ ), with trial sky position $\hat{\Omega}$ and true source position $\hat{\Omega}_{s}$. In this case the cross SNR is equal to

$$
\begin{aligned}
\rho_{\alpha \beta}^{2} \rightarrow & \frac{2}{N} \sum_{k=0}^{N-1} F_{\alpha}^{+}\left[k, \hat{\Omega}_{s}\right] F_{\beta}^{+}\left[k, \hat{\Omega}_{s}\right] \times \frac{\left|\tilde{h}_{+}[k]\right|^{2}}{\sqrt{S_{\alpha}[k] S_{\beta}[k]}} \\
& \times \cos \Psi_{\alpha \beta}\left(\hat{\Omega}, \hat{\Omega}_{s}\right)
\end{aligned}
$$

(only the real part of $\rho_{\alpha \beta}^{2}$ contributes to the energies), where the phase error is

$$
\begin{aligned}
\Psi_{\alpha \beta}\left(\hat{\Omega}, \hat{\Omega}_{s}\right)= & 2 \pi \frac{f_{s} k}{N}\left(\Delta t_{\alpha}(\hat{\Omega})-\Delta t_{\alpha}\left(\hat{\Omega}_{s}\right)-\Delta t_{\beta}(\hat{\Omega})\right. \\
& \left.+\Delta t_{\beta}\left(\hat{\Omega}_{s}\right)\right) .
\end{aligned}
$$

The cross SNR $\rho_{\alpha \beta}^{2}$ for $\alpha \neq \beta$ is typically of the same order of magnitude as $\rho_{\alpha \alpha}^{2}$, but it is positive or negative depending on the timing error between pairs of detectors. As we test different positions on the sky, the timing errors change and the GWB contributions from the different detectors move in and out of phase. This will produce interference fringes in maps of $E_{\text {null }}, E_{\text {null }}-E_{\text {inc }}$, and $E_{\text {null }} / E_{\text {inc }}$. The location and spacing of these fringes are determined by the dominant frequency of the signal, the true sky position of the source, and the detector geometries and relative locations.

In terms of the SNRs (35) and (36), the expectation values of the lowest moments of $E_{\text {null }}, E_{\text {inc }}$, and $E_{\text {null }}-$ $E_{\text {inc }}$ are

$$
\operatorname{mean}\left(E_{\mathrm{null}}\right)=N(D-r)+\sum_{\alpha=1}^{D} \sum_{\beta=1}^{D} Q_{\alpha \beta} \rho_{\alpha \beta}^{2},
$$




$$
\begin{gathered}
\operatorname{var}\left(E_{\text {null }}\right)=N(D-r)+2 \sum_{\alpha=1}^{D} \sum_{\beta=1}^{D} Q_{\alpha \beta} \rho_{\alpha \beta}^{2}, \\
\operatorname{mean}\left(E_{\mathrm{inc}}\right)=N(D-r)+\sum_{\alpha=1}^{D} Q_{\alpha \alpha} \rho_{\alpha \alpha}^{2}, \\
\operatorname{var}\left(E_{\mathrm{inc}}\right)=N \sum_{\alpha=1}^{D} Q_{\alpha \alpha}^{2}+2 \sum_{\alpha=1}^{D} Q_{\alpha \alpha}^{2} \rho_{\alpha \alpha}^{2}, \\
\operatorname{mean}\left(E_{\text {null }}-E_{\mathrm{inc}}\right)=\sum_{\alpha=1}^{D} \sum_{\beta=1}^{D}\left(1-\delta_{\alpha \beta}\right) Q_{\alpha \beta} \rho_{\alpha \beta}^{2},
\end{gathered}
$$

and

$$
\begin{aligned}
\operatorname{var}\left(E_{\text {null }}-E_{\text {inc }}\right)= & N \sum_{\alpha=1}^{D} \sum_{\beta=1}^{D}\left(1-\delta_{\alpha \beta}\right) Q_{\alpha \beta}^{2} \\
& +2 \sum_{\alpha=1}^{D} \sum_{\beta=1}^{D} \sum_{\gamma=1}^{D}\left(1-\delta_{\alpha \beta}\right) \\
& \times\left(1-\delta_{\alpha \gamma}\right) Q_{\alpha \beta} Q_{\alpha \gamma} \rho_{\alpha \gamma}^{2} .
\end{aligned}
$$

We note that the signal enters $E_{\text {inc }}$ only through its SNR $\rho_{\alpha \alpha}^{2}$ in the individual detectors; $E_{\text {inc }}$ does not depend on the structure of the transient signal. As a result, variations in $E_{\text {inc }}$ reflect variations in the network sensitivity due to noise-weighted geometrical factors (the $\boldsymbol{Q}$ ) and do not contain significant information on the signal. By contrast, $E_{\text {null }}-E_{\text {inc }}$ contains only cross terms and shows the interference of the signals measured by the different detectors.

For example, Fig. 4 shows half-sky maps of $E_{\text {null }}, E_{\text {inc }}$ and $E_{\text {null }} / E_{\text {inc }}$ [note that $E_{\text {null }} / E_{\text {inc }}=\left(E_{\text {null }}-E_{\text {inc }}\right) / E_{\text {inc }}+$ 1] for the same GWB and glitch signals used in Fig. 3. The null energy maps for the GWB and the glitch are very similar. The GWB and glitch are constructed to have the same relative time delays and SNRs in the various detectors; as a result, their incoherent energy maps are virtually identical. Removing this signal-independent structure from the null energy makes the signal-dependent structure in the sky maps clearer. In particular, the plot of $E_{\text {null }} / E_{\text {inc }}$ for the GWB shows sharp interference fringes orthogonal to the Hanford-Livingston and Livingston-Virgo baselines (the signal was strongest in Livingston and Virgo in this simulation). The sky location of the GWB signal lies on one of the two intersection points of these interference fringes, so they can be used to locate the source. Such sharp features are not present in the corresponding sky map for the glitch, since the glitch waveforms are not strongly correlated.

We note from (39)-(44) that fractional fluctuations in the energies scale as $N^{-1 / 2}$ for weak signals. Since the signal-dependent structure scales as $\rho_{\alpha \beta}^{2}$, the basic limit of purely local measurements of sky maps scales as $\rho_{\alpha \beta}^{2} / N^{1 / 2}$. This is the same scaling as in excess-power searches [28]. Also, we note that if we restrict our analysis to a frequency range $f=k f_{s} / N \in\left[f_{\min }, f_{\text {max }}\right]$, then $N$ in (39)-(44) becomes the number of frequency bins actually summed over, and $\rho^{2}(35)$ and (36) is to be computed over the same (positive) frequency range. Combining the above, we are able to estimate the signal-to-noise ratio at which the method should become effective. For our simulations we use the three-site Hanford-Livingston-Virgo network, for which $D-r=1$. We restrict to positive frequencies, and use $N=60$ frequency bins. At the true source direction on the sky the difference between the incoherent and null energies has a mean value of approximately $1 / 2 \rho_{\mathrm{rms}}^{2}$, where RMS refers to the average over the three detectors, and the factor of $1 / 2$ arises of the restriction $f>0$. The variance of the measurement is approximately $2 N$. For the difference to be statistically significant we therefore need $\rho_{\text {rms }}^{2}>$ few $\times(8 N)^{1 / 2}$. So, one expects the method to be effective for approximately $\rho_{\text {rms }}>10$.

Finally, we point out that in the high SNR limit the minimum of the null energy $E_{\text {null }}$ occurs at the source location [27], which is useful for solving the inverse problem for bursts. The incoherent energy $E_{\text {inc }}$ is not an extremum at the source location, so measures like $E_{\text {null }} / E_{\text {inc }}$ and $E_{\text {null }}-E_{\text {inc }}$ cannot directly resolve the source location. As noted above, however, the clearer signal-dependent structure in $E_{\text {null }}-E_{\text {inc }}$ and $E_{\text {null }} / E_{\text {inc }}$ compared to $E_{\text {null }}$ may be useful for this purpose. In any case, our consistency test can improve the detection confidence of a gravitationalwave burst and should be regarded as an essential first step in solving the inverse problem.

\section{E. Sensitivity of the method to data calibration}

The derivation of the null-stream combinations described in Sec II B assumed the data from the interferometers to be perfectly calibrated. This means that properly modeled transfer functions of the interferometer responses are applied to the raw data in order to obtain strain measurements. In practice, however, the parameters describing these transfer functions are known with finite accuracy, in turn preventing the null-stream combinations from exactly cancelling the GWB signal at the correct source location. In order to quantify the effect on our method we should note that the magnitude of the residual signal in the nullstream combinations will be proportional to the accuracy by which the calibration parameters are known. For the LIGO detectors, the calibration parameters are known to better than $10 \%$ [44]. Preliminary studies indicate our consistency test is robust against calibration errors of this size; these effects will be studied in more detail in a future article.

\section{F. Implementation}

Our null-stream-based analysis has been implemented as a publicly available MATLAB package, XPIPELINE [45]. For a specific detector network and event time, XPIPELINE reads the appropriate data from frame files [46] (the stan- 

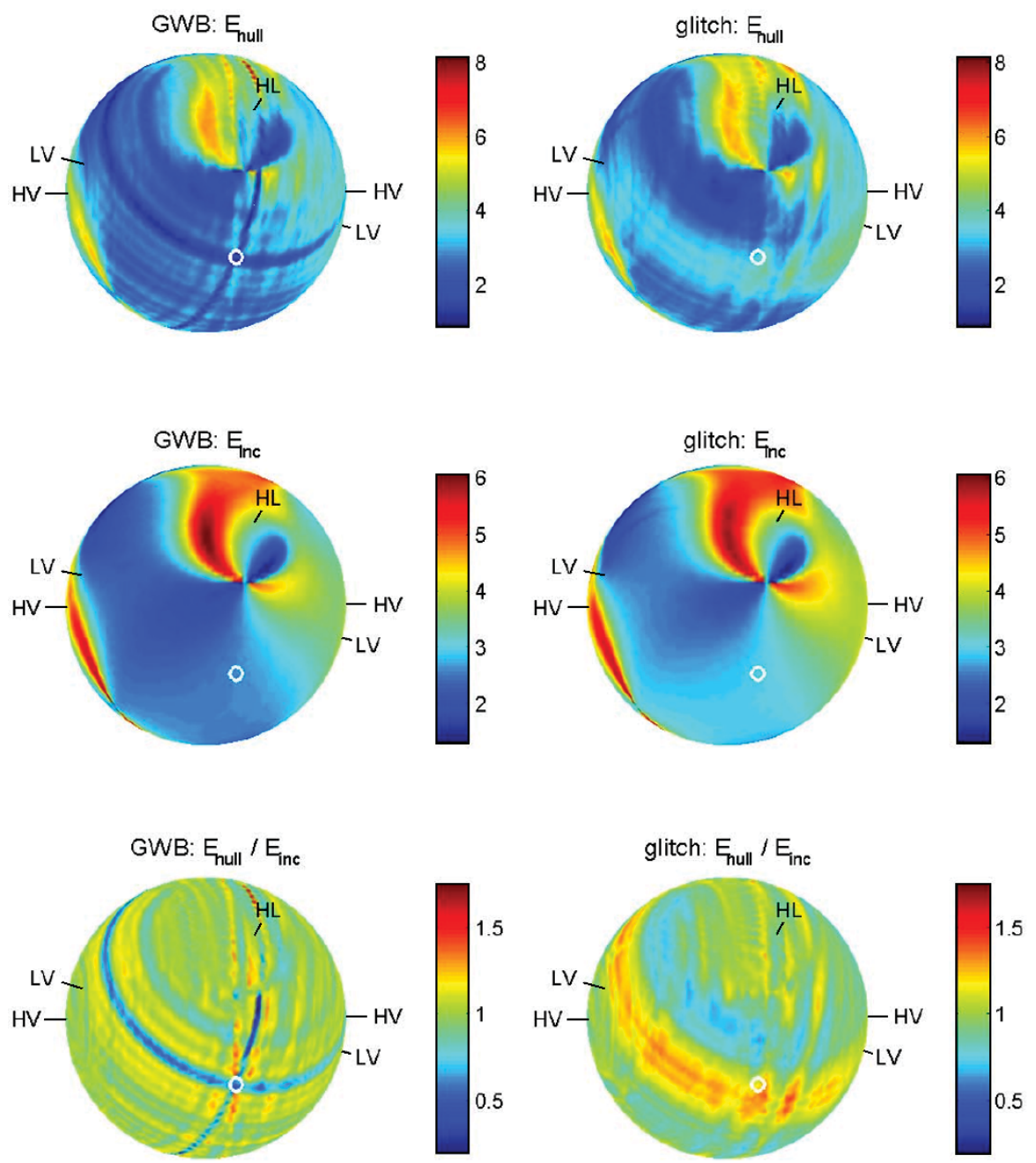

FIG. 4 (color). Sample sky hemisphere maps, normalized to $E /(N(D-r))$, for the same GWB and glitch signals used in Fig. 3. The plots on the left are for a GWB with rms SNR of 20 in the three detectors. Those on the right are for a glitch with the same relative time delays and signal energies in each detector as the GWB. (Note that these two events are indistinguishable to an incoherent analysis.) The network consists of the LIGO-Hanford (H) and LIGO-Livingston (L) 4 km detectors and Virgo (V). The null energy map (top) shows interference fringes due to the transient signal, as well as structure due to the network geometry. They are very similar for the GWB and the glitch. The incoherent energy map (middle) is constructed from the autocorrelations of the individual detector data streams and reflects variations in the network sensitivity over the sky. It is virtually identical for the GWB and the glitch because the two events have the same relative time delays and SNRs. Dividing out the incoherent energy from the null energy (bottom) removes this structure associated with the network geometry, making the signal-dependent structure clearer. The GWB hemisphere map shows sharp interference fringes (blue and red rings)where the time delays along the $\mathrm{H}-\mathrm{L}, \mathrm{L}-\mathrm{V}$, and $\mathrm{H}-\mathrm{V}$ baselines match those of the source location; this is a realization of the sketch in Fig. 1. There is little of such structure in the glitch hemisphere map. The source location in each map is marked by a circle.

dard format for storing data from gravitational-wave detectors), optionally injects GWB signals and/or glitches, whitens the data, computes the null-stream coefficient matrix $\boldsymbol{A}$ for each specified sky direction and frequency, computes the time shifts for each direction, steps through data in overlapping blocks of user-specified duration, timeshifts the data to the nearest sample, Fourier transforms it, completes the time shift with a phase rotation, forms the null stream in the frequency domain, sums the power in user-specified frequency bands, and records the null and incoherent energies for each time-frequency band and direction. XPIPELINE runs in approximately $1 / 100$ real time. For example, the analysis of the $10^{4}$ simulated events used to produce Figs. 6-11 took approximately $16 \mathrm{~h}$ on 4 Intel Pentium 4, 2.66 GHz computers. This makes our nullstream-based consistency test feasible as a follow-up test in GWB searches.

\section{SIMULATIONS}

\section{A. Network and signal types}

To test the efficacy of our statistical test in discriminating GWBs from noise glitches in the $\left(E_{\text {null }}, E_{\text {inc }}\right)$ space, we 
need to select a detector network, a population of GWBs, and a population of glitches.

We elect to simulate a network consisting of the LIGOHanford and LIGO-Livingston $4 \mathrm{~km}$ interferometers (" $\mathrm{H}$ " and "L") and a third identical instrument at the Virgo site ("V"). For the sake of simplicity we neglect both the Hanford $2 \mathrm{~km}$ interferometer and the differences between the LIGO and Virgo design sensitivities. The locations $\vec{r}_{\alpha}$ and orientations (which determine $F^{+}, F^{\times}$) of the interferometers are taken from $[28,47]$. The calibrated stationary background noise $n_{\alpha}[j]$ for $\mathrm{H}$ and $\mathrm{L}$ are taken from a standard $24 \mathrm{~h}$ reference simulation [48]. The background noise for $\mathrm{V}$ is taken from the Hanford simulation with a $2 \mathrm{~s}$ time shift, which is much larger than the time scales of the signals used in this analysis.

The next step is to select the GWB and glitch waveforms. To simulate a glitch, we need three waveforms (one for each detector) that are not strongly correlated. To simulate GWBs we wish to use waveforms that are motivated by astrophysical considerations. Our consistency test is ultimately based on the fact that for a GWB the signal seen in each detector is correlated in a particular way, whereas for a glitch the signals generally will not be correlated. In order to show that our test does not rely on any fundamental difference between "typical" GWB waveforms and typical glitch waveforms, we elect to use the same set of waveforms for simulating GWBs and glitches. We select three representative waveforms from the Dimmelmeier-Font-Mueller ("DFM") catalog [15] of type II core-collapse supernovae. Specifically, we choose the A1B3G3 "regular collapse" waveform, the A1B3G5 "rapid collapse" waveform, and the A3B4G2 "multiple bounce" waveform. Figure 5 shows the time series and power spectra of these waveforms. As we shall see below, these three waveforms have moderately low but nonzero cross correlations.

To simulate a gravitational-wave signal, one of the three waveforms is randomly selected and added into the data stream from each interferometer, with time delays and scaling appropriate for some choice of polarization angle and location on the sky. To simulate a glitch we follow the same procedure, except that a different waveform is selected for each detector. The scaling and time delays proceed as for a GWB. This population of glitches has the property that it would pass any incoherent test, as the arrival times, power distribution, and even frequency bands are consistent with those of true gravitational-wave signals. We stress that, though not a realistic glitch population, this provides us with examples of the kind of pathological glitches that cannot be dismissed by per-detector methods.

\section{B. Analysis parameters}

The main tunable parameters in our analysis are the time and frequency bands and the sky positions to test. Ideally the integration time and frequency band should be matched to the signal being tested, to maximize the signal-to-noise ratios $\rho_{\alpha \alpha}^{2}$ and minimize the background noise contribution $N(D-r)$. We choose an integration length of $1 / 16 \mathrm{~s}$, which is the smallest power of 2 larger than the durations of the three sample waveforms tested. We overlap consecutive data segments by $50 \%$ to minimize the loss of signalto-noise when a signal overlaps the edge of a data segment. We use a single frequency band [49] of [64 1024] Hz. The upper frequency limit is set by the highest frequency at which our target signals have significant power compared to the noise curve; see Fig. 5. The lower limit is set at $64 \mathrm{~Hz}$ because the actual noise level in current detectors is larger than the design noise below this cutoff [50].

Once the locations of the detectors and the upper bound on frequencies involved in the analysis are known, a set of directions $\{\hat{\Omega}\}$ covering the sky may be produced. Both the projection matrix $\mathbf{Q}(26)$ and the time delays $\Delta t_{\alpha}$ (2) vary with angle, but the effect of $\Delta t_{\alpha}$ on the cross correlation terms occurs on a smaller angular scale for most of the sky. A simple criteria then is to cover the sky with a maximum angular mismatch defined by the longest detector baseline and the maximum frequency. As with all template placement problems we have some freedom in how to produce
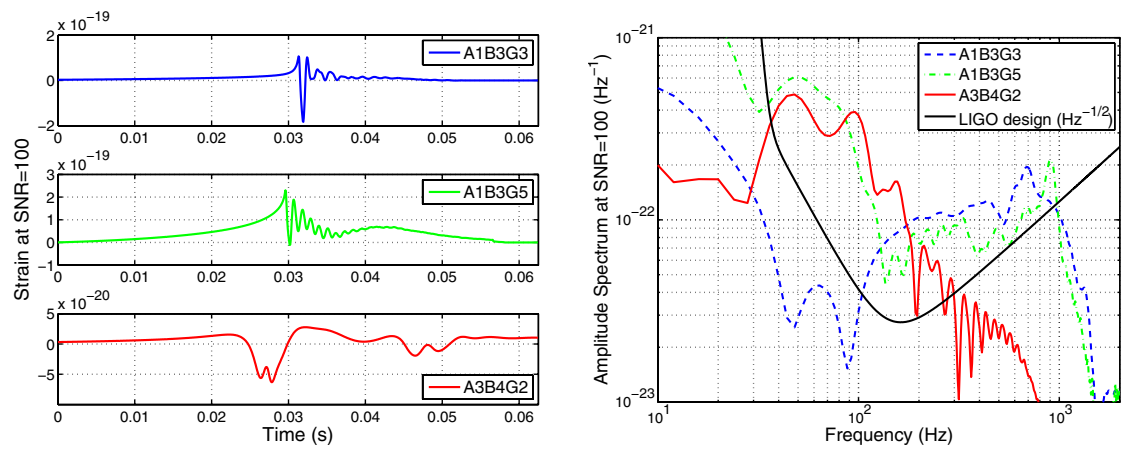

FIG. 5 (color online). Dimmelmeier-Font-Mueller waveforms used to simulate GWBs and glitches in our analysis. Left plot: time-series waveforms scaled to SNR $=100$ for optimal orientation. (The linear trend present in the original waveforms has been removed.) Right plot: spectra with a $1 \mathrm{~Hz}$ resolution; shown for comparison is the LIGO design noise curve used for both LIGO and Virgo detectors. 
this set and chose a somewhat suboptimal but simple set of directions - a grid of approximately $10^{4}$ points uniformly distributed in $\theta$ and $\phi \sin \theta$.

\section{Waveform normalization}

The DFM waveforms are linearly polarized, which means that the two polarizations are linearly dependent and so can be written in the form

$$
\begin{aligned}
& h_{+}(t)=\cos \left(2 \psi_{s}\right) h(t), \\
& h_{\times}(t)=\sin \left(2 \psi_{s}\right) h(t),
\end{aligned}
$$

where $\psi_{s}$ is the polarization angle. As a result, the strain signal $g_{\alpha}$ in detector $\alpha$ is

$$
g_{\alpha}(t)=\left(F_{\alpha}^{+}\left(\hat{\Omega}_{s}\right) \cos 2 \psi_{s}+F_{\alpha}^{\times}\left(\hat{\Omega}_{s}\right) \sin 2 \psi_{s}\right) h(t) .
$$

These waveforms are detrended and normalized against the interferometer noise curve so that $\rho_{\alpha \alpha}^{2}=1$ (36) for optimal orientation [the case $F_{\alpha}^{+} \cos 2 \psi_{s}+F_{\alpha}^{\times} \sin 2 \psi_{s}=1$, so that $g_{\alpha}(t)=h(t)$ ]; i.e., we define the normalization of $h(t)$ so that

$$
\sum_{k=0}^{N-1} \frac{|\tilde{h}[k]|^{2}}{\frac{N}{2} S[k]}=1
$$

(Recall that our detectors have identical noise spectra, so $S_{\alpha}[k]=S_{\beta}[k] \equiv S[k]$.) The cross correlations (35) of the waveforms depend on their relative time or phase shift; with this normalization the maximum cross correlations over all shifts for co-aligned detectors are

$$
\max _{\Psi}\left\{\rho_{\alpha \beta}^{2}\right\}= \begin{cases}0.58 & (\mathrm{~A} 1 \mathrm{~B} 3 \mathrm{G} 3-\mathrm{A} 1 \mathrm{~B} 3 \mathrm{G} 5) \\ 0.26 & (\mathrm{~A} 1 \mathrm{~B} 3 \mathrm{G} 3-\mathrm{A} 3 \mathrm{~B} 4 \mathrm{G} 2) \\ 0.50 & (\mathrm{~A} 1 \mathrm{~B} 3 \mathrm{G} 5-\mathrm{A} 3 \mathrm{~B} 4 \mathrm{G} 2)\end{cases}
$$

For comparison, typical cross correlation values for Gaussian noise in our time-frequency band are 0.15-0.2.

To simulate a gravitational-wave signal, one of the three waveforms A1B3G3, A1B3G5, or A3B4G2 is randomly selected. An isotropically distributed random sky direction $\hat{\Omega}_{s}$ and a uniformly distributed polarization angle $\psi_{s}$ are chosen. For each detector $\alpha$, the discrete catalog waveform $h(t)$ is time-shifted by $\Delta t_{\alpha}\left(\hat{\Omega}_{s}\right)(2)$ and resampled to match $n_{\alpha}[j]$. The waveform is then scaled by the antenna response as in (47) to give $g_{\alpha}$.

To characterize the efficacy of our consistency test as a function of the signal strength, we choose to simulate populations of candidates with the same measured signalto-noise ratios; i.e., the signals are scaled so as to deliver a fixed total SNR to the network. This simulates candidates near a detection threshold, rather than (for example) a physical population of standard candles. With the normalization (48) and (47) one finds

$$
\begin{aligned}
\frac{1}{D} \sum_{\alpha=1}^{D} \rho_{\alpha \alpha}^{2} & =\frac{1}{D} \sum_{\alpha=1}^{D} \sum_{k=0}^{N-1} \frac{\left|g_{\alpha}[k]\right|^{2}}{\frac{N}{2} S[k]} \\
& =\frac{1}{D} \sum_{\alpha=1}^{D}\left(F_{\alpha}^{+}\left(\hat{\Omega}_{s}\right) \cos 2 \psi_{s}+F_{\alpha}^{\times}\left(\hat{\Omega}_{s}\right) \sin 2 \psi_{s}\right)^{2} .
\end{aligned}
$$

To fix the SNR in the detectors to some rms value $\rho_{\text {rms }}$, we apply the further normalization

$$
g_{\alpha}(t) \rightarrow \frac{g_{\alpha}(t) \rho_{\mathrm{rms}}}{\sqrt{\frac{1}{D} \sum_{\beta=1}^{D}\left(F_{\beta}^{+}\left(\hat{\Omega}_{s}\right) \cos 2 \psi_{s}+F_{\beta}^{\times}\left(\hat{\Omega}_{s}\right) \sin 2 \psi_{s}\right)^{2}}} .
$$

With this scaling we find

$$
\sqrt{\frac{1}{D} \sum_{\alpha=1}^{D} \rho_{\alpha \alpha}^{2}}=\rho_{\text {rms }}
$$

To simulate a population of glitches, the same process was followed with the sole exception that a different DFM waveform was selected for each detector. We applied the same scaling and time delays as for a GWB. This population of glitches has the property that it would pass any incoherent test, as its arrival times and power distribution are consistent with those of true gravitational-wave signals. We reiterate that, though not a realistic glitch population, this provides us with examples of the kind of pathological glitches that cannot be dismissed by per-detector methods.

\section{Analysis procedure}

After the signal has been added to the background noise, the data are whitened to produce $d_{\mathrm{w} \alpha}[j]$. The whitening algorithm is trained on a $16 \mathrm{~s}$ block of data that does not include the signal. With a known set of trial sky positions $\{\hat{\Omega}\}$ and measured power spectra $S_{\alpha}(f), \mathbf{Q}$ can be computed for each direction and resolvable frequency.

For each direction on the sky, overlapping segments of data are considered sequentially, the length of the segments depending on the time scales of the signal under consideration (here chosen as $1 / 16 \mathrm{~s}$ ). To perform the time shifts, the segments are extracted from the closest integer samples in the time domain and then transformed to the Fourier domain where the remaining part of the time shift is performed by applying phase shifts. The null and incoherent energies are then computed and recorded for that direction on the sky.

For each simulation we select the two directions for which the transient shows the most correlation according to two different criteria, as well as the direction with the minimum null energy:

(1) $\min \left(E_{\text {null }} / E_{\text {inc }}\right)$ : Measures the angular distance away from the diagonal in a scatter plot of $E_{\text {inc }}$ vs $E_{\text {null }}$ (see Fig. 3). Physically it represents the largest 
fraction of energy cancelled in forming the null stream.

(2) $\min \left(E_{\text {null }}-E_{\text {inc }}\right)$ : Measures the linear distance away from the diagonal in a scatter plot of $E_{\text {inc }}$ vs $E_{\text {null }}$ (see Fig. 3). Physically it represents the largest amount of energy cancelled in forming the null stream.

(3) $\min \left(E_{\text {null }}\right)$ : Calculated for comparison against the other two statistics and for estimating the sky location.

Figures 6-8 show scatter plots of $E_{\text {inc }}$ vs $E_{\text {null }}$ for the sky locations picked by these three criteria for a population of $10^{4}$ simulated signals. The signal population consists of $10^{3} \mathrm{GWBs}$ and $10^{3}$ glitches at each of five different signalto-noise ratios, $\rho_{\text {rms }}=5,10,20,50,100$. We make independent random draws for sky position and polarization for each GWB or glitch tested.

Note that there is a significant difference in the distributions of signals and glitches using any of the measures $\min \left(E_{\text {null }}-E_{\text {inc }}\right), \min \left(E_{\text {null }} / E_{\text {inc }}\right)$, or $\min \left(E_{\text {null }}\right)$. For both GWBs and glitches there are directions on the sky for

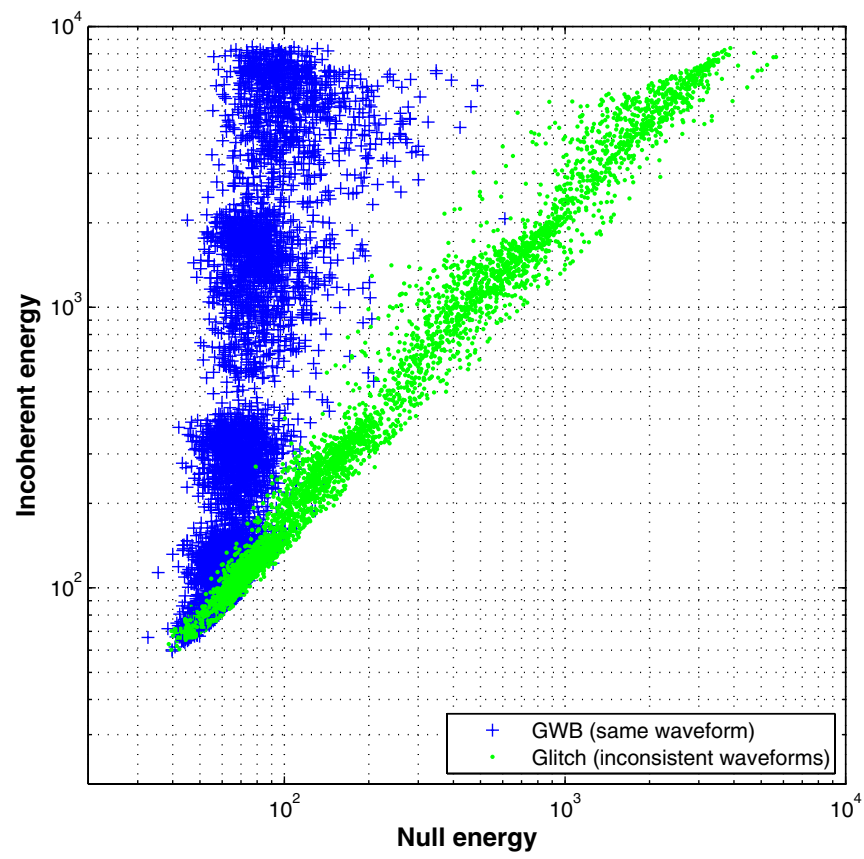

FIG. 6 (color online). Scatter plot of the null and incoherent energies for the most correlated direction on the sky, defined as the direction of $\min \left(E_{\text {null }} / E_{\text {inc }}\right)$ (i.e., the upper left limit of Fig. 3), for glitches and GWBs of various signal-to-noise ratios. Note that as the signal-to-noise ratio increases (higher $E_{\text {inc }}$ ) the GWB and glitch distributions separate, with the GWBs remaining at relatively low null energies and the glitches having comparable null and incoherent energies. The clumping is due to the 5 distinct rms SNRs used in our simulations: 5, 10, 20, 50, 100. The null energies for some of the GWB simulations are greater than that expected (60) from the number of degrees of freedom because we are selecting sky positions from the minimum of $E_{\text {null }} / E_{\text {inc }}$, not from the minimum of $E_{\text {null }}$. which the null energy is low. However, for glitches these occur exclusively at low incoherent energies. For signals there exist directions with low null energy at larger incoherent energies. This restriction is strongest when measuring correlations using $\min \left(E_{\text {null }} / E_{\text {inc }}\right)$ (Fig. 6). For $\min \left(E_{\text {null }}-E_{\text {inc }}\right)($ Fig. 7) there are a small but noticeable fraction of GWBs that overlap the glitch population and so are indistinguishable from glitches. When using $\min \left(E_{\text {null }}\right)$ only (Fig. 8) a significant fraction of the glitches have a sky location with low null energy and overlap the population of weak GWBs.

As the SNR increases, the populations become distinct. We can see this also in Figs. 9-11, which show the receiver-operator characteristic (ROC) curves for the performance of our various statistics in discriminating the two populations. These curves show the fraction of GWBs that pass the consistency test versus the fraction of glitches that pass, at fixed SNR. At total energies corresponding to $\rho_{\text {rms }} \sim 10-20$ or greater we can detect most of a population of gravitational waves and reject essentially all of a popu-

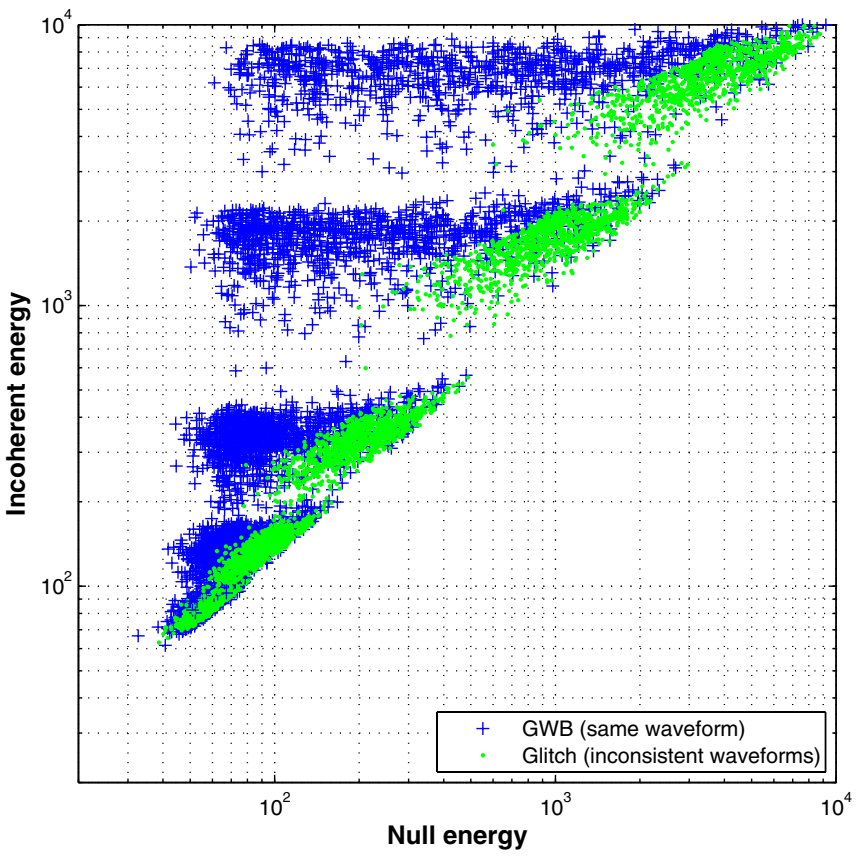

FIG. 7 (color online). Scatter plot of the null and incoherent energies for the most correlated direction on the sky, defined as the direction of $\min \left(E_{\text {null }}-E_{\text {inc }}\right)$ (i.e., the upper left limit of Fig. 3), for glitches and GWBs of various signal-to-noise ratios. Note that as the signal-to-noise ratio increases (higher $E_{\text {inc }}$ ) the GWB and glitch distributions separate, with the GWBs remaining at relatively low null energies and the glitches having comparable null and incoherent energies. The clumping into horizontal bands is due to the five distinct rms SNRs used in our simulations: 5, 10, 20, 50, 100. The null energies for many of the GWB simulations are greater than that expected (60) from the number of degrees of freedom because we are selecting sky positions from the minimum of $E_{\text {null }}-E_{\text {inc }}$, not from the minimum of $E_{\text {null }}$. 


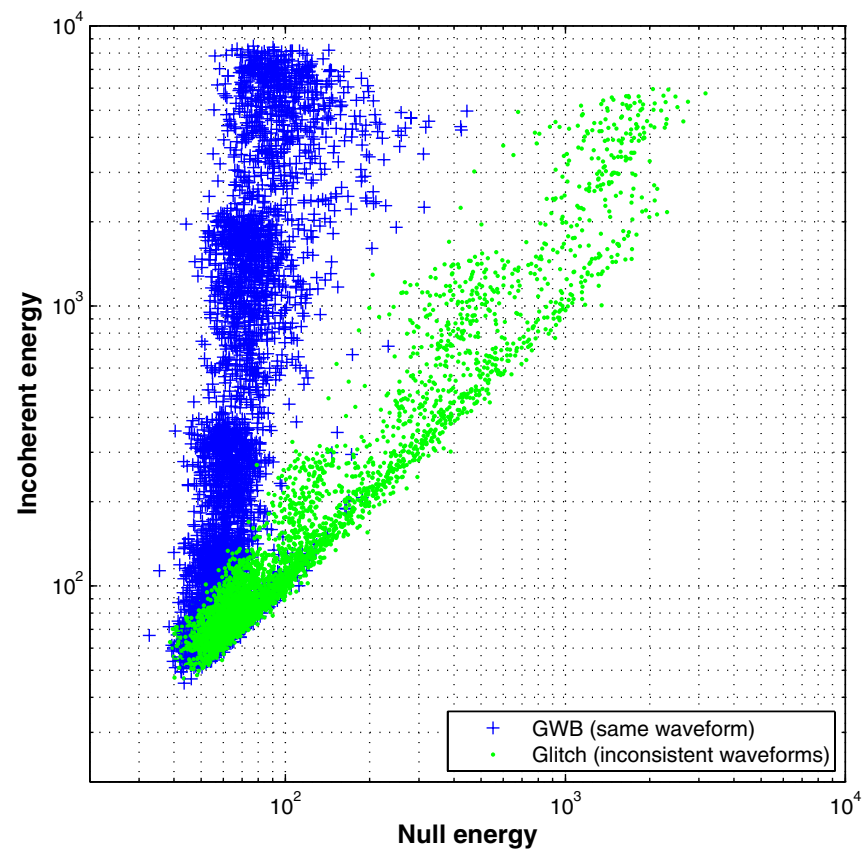

FIG. 8 (color online). Scatter plot of the null and incoherent energies for the best-fit direction on the sky, defined as the direction of $\min \left(E_{\text {null }}\right)$ (i.e., the left-most point in Fig. 3) for glitches and GWBs of various signal-to-noise ratios. Note that as the signal-to-noise ratio increases (higher $E_{\text {inc }}$ ) the GWB and glitch distributions separate, with the GWBs remaining at relatively low null energies and the glitches having comparable null and incoherent energies. The clumping is due to the 5 distinct rms SNRs used in our simulations: 5, 10, 20, 50, 100. Note that a large fraction of the glitch signals produce low null energies for some sky positions. This means that GWBs and glitches are not distinguishable using only the null energy.

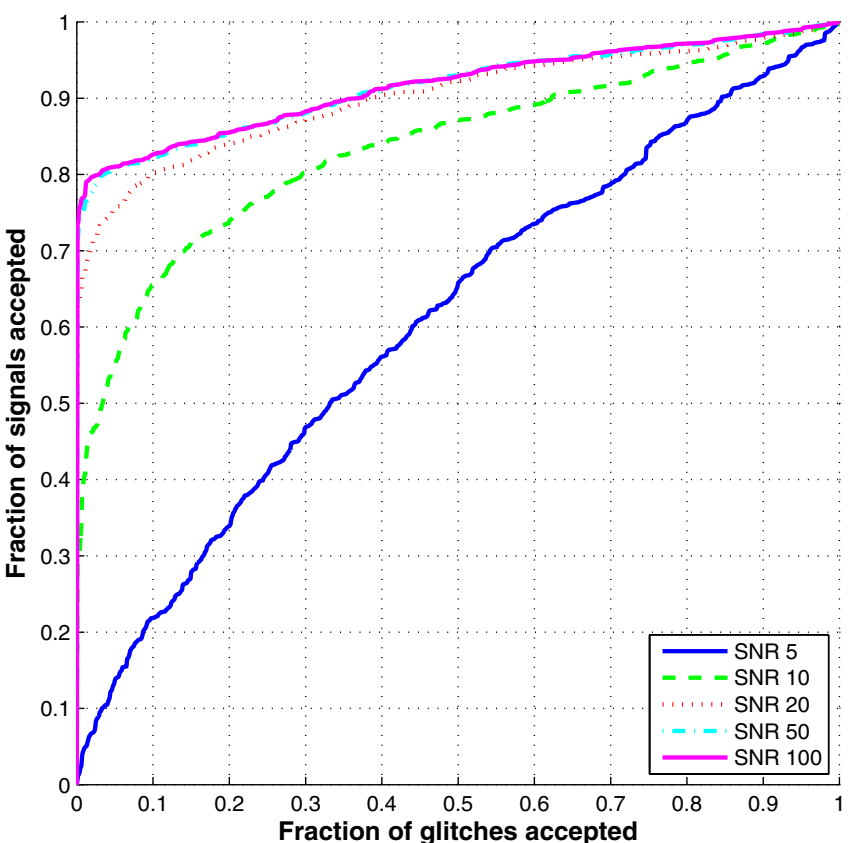

FIG. 10 (color online). ROC for $\min \left(E_{\text {null }}-E_{\text {inc }}\right)$ as a statistic for distinguishing GWBs from noise glitches. The ROC curve value is given by the fraction of GWBs (true acceptance) and glitches (false acceptance) of given rms SNR falling to the left of a line of constant $E_{\text {null }}-E_{\text {inc }}$ in Fig. 7. The rapid rise of the curves at low false acceptance is indicative of the ability of the method to confidently distinguish a significant portion of GWB signals from the glitch population. This is not as powerful a statistic as $\min \left(E_{\text {null }} / E_{\text {inc }}\right)$ (see Fig. 9).

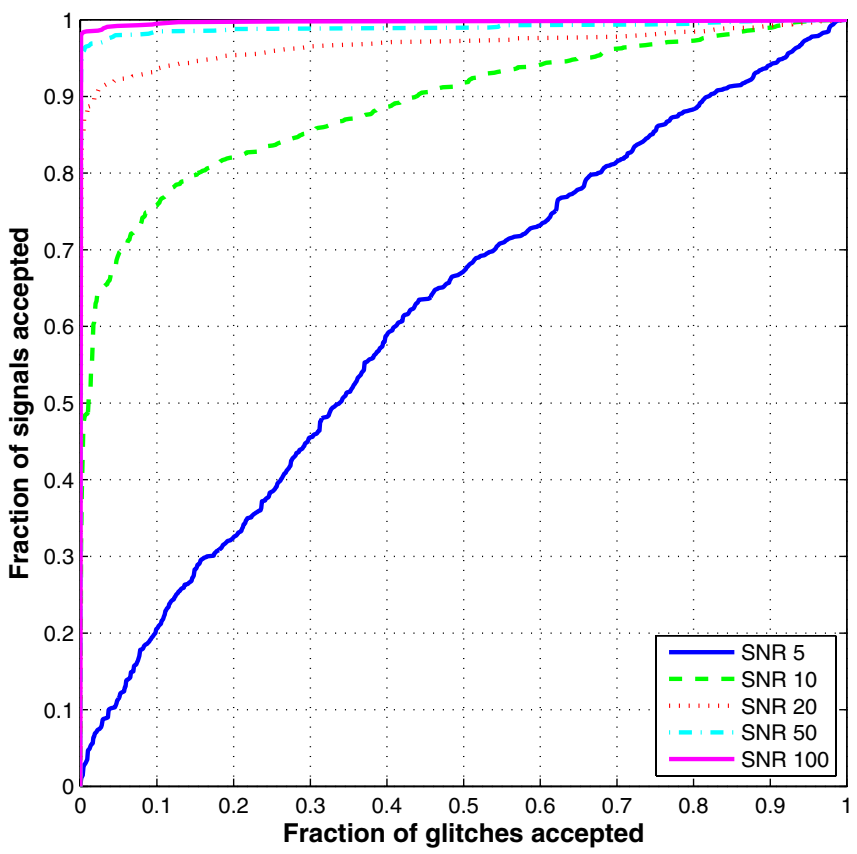

FIG. 9 (color online). ROC plot for $\min \left(E_{\text {null }} / E_{\text {inc }}\right)$ as a statistic for distinguishing GWBs from noise glitches. The ROC curve value is given by the fraction of GWBs (true acceptance) and glitches (false acceptance) of given rms SNR falling to the left of a line of constant $E_{\text {null }} / E_{\text {inc }}$ (a diagonal line) in Fig. 6. The rapid rise of the curves at low false acceptance is indicative of the ability of the method to confidently distinguish a significant portion of GWB signals from the glitch population. 


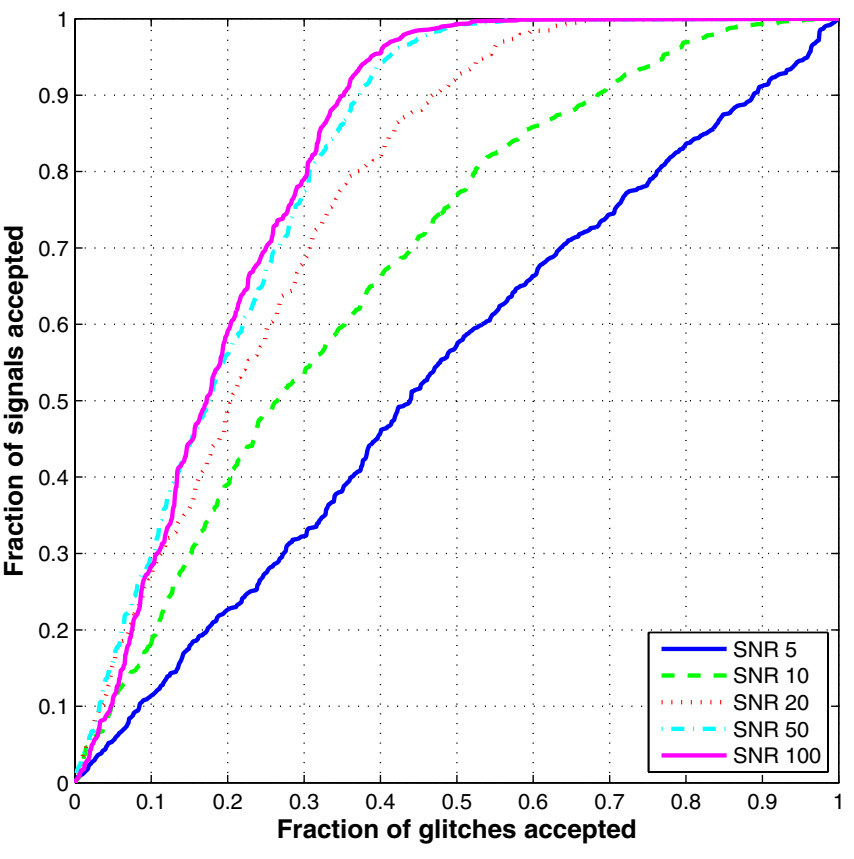

FIG. 11 (color online). ROC plot for $\min \left(E_{\text {null }}\right)$ as a statistic for distinguishing GWBs from noise glitches. The ROC curve value is given by the fraction of GWBs (true acceptance) and glitches (false acceptance) of given rms SNR falling to the left of a line of constant $E_{\text {null }}$ (a vertical line) in Fig. 8. The lower slope of the curves at low acceptance is due to the fact that many glitches have sky positions for which the null energy is small, even when the SNR of the glitch is high. The null energy alone is therefore not very effective for confidently distinguishing GWBs from noise glitches. While one could chose a threshold that varies with $E_{\text {inc }}$ to get better performance, $\min \left(E_{\text {null }}\right)$ is still not as powerful a statistic as $\min \left(E_{\text {null }} / E_{\text {inc }}\right)$.

lation of semicorrelated glitches. The rejected gravitational waves are those that are weak in at least one detector, or equivalently produce little correlation. An SNR of $\sim 10-20$ is comparable to the level at which current LIGO burst detection algorithms have a detection efficiency of $50 \%$ or greater [24]. Furthermore, glitches of this size are common in real detectors, indicating that our test will in fact be useful with present detector networks.

For $\rho_{\text {rms }}=5$ or lower, the glitch and GWB populations are not distinct. This is because the null stream enforces waveform consistency only when there is excess energy to suppress.

\section{CONCLUSIONS AND FUTURE DIRECTIONS}

In this article we have introduced an extension of the Gürsel-Tinto null-stream technique that allows one to make robust tests to distinguish between GWB signals and coincident noise glitches. This technique is based on comparing the energy in the null stream to that expected if the signals in the various detectors are uncorrelated and does not require any a priori knowledge of the GWB or glitch waveforms. We applied this technique to the case of the LIGO-Virgo 3-detector network at design sensitivity and quantified the ability of three different measures of correlation to distinguish true GWBs from coincident noise glitches. For the best-performing measure, the ratio of null energy to incoherent energy, we found that gravitationalwave bursts of SNR 10-20 or greater can be distinguished from glitches of comparable SNRs that are injected in the data with the same time delays but are different in the three detectors' data. For example, with the GWB and glitch populations tested, we found that $90 \%$ of glitches can be rejected while accepting $94 \%$ of SNR 20 GWBs and 76\% of SNR 10 GWBs. Furthermore, we stress that the glitch population tested was pathological in the sense that they were constructed to have time delays and amplitudes consistent with a GWB. Hence, the performance of the consistency test may be even better with real detector data. This consistency test is therefore a promising technique for rejecting noise coincidences and increasing detection confidence in GWB searches.

The development of coherent analysis techniques for GWB detection is still at an early stage, and much further research can be done. In this section we briefly outline some of the directions of current and future work. These can be divided roughly into applications of the existing consistency test to more general networks and signals and extensions and improvements to the algorithm.

We will systematically study a larger variety of waveforms than the small set considered in the paper. These should include two-polarization GWBs. (The supernova waveforms used here are linearly polarized.) This wider set may include various supernova catalogs [14-16] and approximate waveforms for black-hole binary coalescence (see for example [7-11]). The latter are particularly important, since it is quite plausible that black-hole binaries will be the first transient signals to be detected. Preliminary tests with Lazarus black-hole merger waveforms [51] indicate that our test is as effective as for the linearly polarized waveforms tested here.

Another near-term goal is to apply our modified nullstream test to other networks, such as those with four and five detectors. A fourth nonaligned detector will reduce the fraction of sky over which only two detectors have significant sensitivity [43], increasing the strength of the test. An additional aligned detector, such as the two-kilometer detector at LIGO-Hanford, would also provide a second null stream without extra sky coverage. Lazzarini et al. [52] have demonstrated how the output of the two LIGOHanford detectors can be combined to form a single pseudodetector with greater sensitivity than either. The difference between the detector outputs is also a null stream, effectively a detector with zero antenna response, which can be employed in our consistency test. This is a computationally cheap test since the $\mathrm{H} 1-\mathrm{H} 2$ null stream is independent of the sky position, providing the basis of a simple hierarchical analysis scheme. 
We also plan to test the power of our consistency test on real data, with real noise transients. We should note that the artificial noise coincidences studied in this work are pathological in the sense that they are injected with time delays and amplitude responses consistent with actual sky positions and waveforms that are identical (in individual detectors) to GWBs. Although we would not expect noise in actual detectors to be so pathological, it is important to characterize the robustness of our technique for real data, and this was the motivation for our implementation.

Another important aspect of real data is that their calibration could be inaccurate, implying an imperfect cancellation of the GWB by the null stream. We will study quantitatively the effects of realistic calibration errors on the effectiveness of the null-stream technique (see also the study by Ajith et al. [53]).

A first improvement is to optimize the algorithm for implementing our test. An optimal identification of the integration time and frequency band over which the null and incoherent energies are calculated will improve the effectiveness of the test by minimizing the amount of noise included.

An important augmentation of the coherent analysis is improved techniques for determining the sky location of the source. Current efforts estimate the sky position as the extremum in a sky map of the null stream or some likelihood statistic [27,29]. For example, Gürsel and Tinto [27] demonstrated that locating the minimum of the null energy allows one to determine the direction to the source of a kilohertz GWB with high accuracy for typical SNRs of 40-60 (converted to our $\rho_{\text {rms }}$ ). However, as we have seen, sky maps exhibit structure which is a combination of the network geometry and the signal waveform. For example, linearly polarized signals produce interference fringes in the sky map. These fringes appear as rings of fixed time delay with respect to the various detector baselines. A global analysis which takes account of this structure could in principle average over local noise fluctuations in the sky map to achieve an improved pointing accuracy for weaker signals.

We will further investigate Bayesian interpretations and formulations of the null-stream technique and compare the effectiveness of this approach against the procedure presented in this work. We will also investigate the possibility of incorporating additional tests such as distribution-free (nonparametric) correlation tests. These could prove valuable when analyzing real data since they enforce known statistics even when the noise in the data follows an arbitrary noise distribution function.

\section{ACKNOWLEDGMENTS}

The authors wish to thank Malik Rakhmanov for useful comments. This work was performed under partial funding from the following NSF Grants No. PHY-0107417, No. 0140369, No. 0239735, No. 0244902, No. 0300609, and No. INT-0138459. A. Searle was supported by the Australian Research Council and the LIGO Visitors Program. L. Stein was supported in part by an NSF REU Site grant. Simulations were performed on the Australian Partnership for Advanced Computing's National Facility under the Merit Allocation Scheme. For M. Tinto, the research was also performed at the Jet Propulsion Laboratory, California Institute of Technology, under contract with the National Aeronautics and Space Administration. This document has been assigned LIGO Laboratory document number LIGO-P060009-02-E.

\section{APPENDIX A: MINIMUM-VARIANCE WAVEFORM RECONSTRUCTION}

In this section we derive explicit expressions for the two amplitude components of the wave, $h_{+}$and $h_{\times}$, as optimally reconstructed as linear combinations of the detector data. This is a generalization of the technique used by Gürsel and Tinto [27] and was first derived by Flanagan and Hughes [6]. We will follow the very elegant derivation by Rakhmanov [32].

We will work exclusively in the Fourier domain. For notational convenience, we will drop explicit references to the frequency and sky position and also drop the tildes used to denote Fourier domain quantities. We will also assume that the whitened data streams have been time-shifted before these combinations are constructed and will simply write, for example, $d_{\mathrm{w} \alpha}$ for $\tilde{d}_{\mathrm{w} \alpha}\left[f_{i}\right] \mathrm{e}^{i 2 \pi f_{i} \Delta t_{\alpha}(\theta, \phi)}$.

Our goal is to construct the linear combination of the $d_{\mathrm{w} \alpha}$ which is the best fit to the unknown $h_{+}$and $h_{\times}$in the least-squares sense. That is, we seek

$$
\hat{\boldsymbol{h}}=\left[\begin{array}{l}
\hat{h}_{+} \\
\hat{h}_{\times}
\end{array}\right]
$$

such that

$$
0=\left.\frac{d}{d \boldsymbol{h}^{T}}\left(\boldsymbol{d}_{\mathrm{w}}-\boldsymbol{F}_{\mathrm{w}} \boldsymbol{h}\right)^{T}\left(\boldsymbol{d}_{\mathrm{w}}-\boldsymbol{F}_{\mathrm{w}} \boldsymbol{h}\right)\right|_{\boldsymbol{h}=\hat{\boldsymbol{h}}} .
$$

(Note that since we use whitened data, the least-squares fit is also the maximum-likelihood fit in the presence of Gaussian noise.) The solution is

$$
\hat{\boldsymbol{h}}=\boldsymbol{F}_{\mathrm{MP}}^{-1} \boldsymbol{d}_{\mathrm{w}},
$$

where

$$
\boldsymbol{F}_{\mathrm{MP}}^{-1} \equiv\left(\boldsymbol{F}_{\mathrm{w}}{ }^{T} \boldsymbol{F}_{\mathrm{w}}\right)^{-1} \boldsymbol{F}_{\mathrm{w}}{ }^{T} .
$$

As pointed out by Rakhmanov [32], the waveform reconstruction matrix $\boldsymbol{F}_{\mathrm{MP}}^{-1}$ is the pseudoinverse or MoorePenrose inverse of the antenna response matrix $\boldsymbol{F}$ :

$$
\boldsymbol{F}_{\mathrm{MP}}^{-1} \boldsymbol{F}=\boldsymbol{I}_{2 \times 2} .
$$

It is worth noting the explict form of the reconstructed waveforms in terms of $\boldsymbol{F}_{\mathrm{w}}^{+}$and $\boldsymbol{F}_{\mathrm{w}}^{\times}$. Denoting the inner products of these vectors with each other by 


$$
\begin{aligned}
& \boldsymbol{F}_{++}=\boldsymbol{F}_{\mathrm{w}}^{+} \cdot \boldsymbol{F}_{\mathrm{w}}^{+}, \\
& \boldsymbol{F}_{+\times}=\boldsymbol{F}_{\mathrm{w}}^{+} \cdot \boldsymbol{F}_{\mathrm{w}}^{\times}, \\
& \boldsymbol{F}_{\times \times}=\boldsymbol{F}_{\mathrm{w}}^{\times} \cdot \boldsymbol{F}_{\mathrm{w}}^{\times},
\end{aligned}
$$

we have

$$
\begin{aligned}
{\left[\begin{array}{c}
\hat{h}_{+} \\
\hat{h}_{\times}
\end{array}\right]=} & \frac{1}{\boldsymbol{F}_{++} \boldsymbol{F}_{\times \times}-\boldsymbol{F}_{+\times}{ }^{2}}\left[\begin{array}{cc}
\boldsymbol{F}_{\times \times} & -\boldsymbol{F}_{+\times} \\
-\boldsymbol{F}_{+\times} & \boldsymbol{F}_{++}
\end{array}\right] \\
& \times\left[\begin{array}{c}
\boldsymbol{F}_{\mathrm{W}}^{+} \cdot \boldsymbol{d}_{\mathrm{w}} \\
\boldsymbol{F}_{\mathrm{w}}^{\times} \cdot \boldsymbol{d}_{\mathrm{w}}
\end{array}\right] .
\end{aligned}
$$

Note that the weighting of a particular detector $d_{\alpha}$ vanishes if $F_{\mathrm{w} \alpha}^{+}=0=F_{\mathrm{w} \alpha}^{\times}$, which occurs if detector $d_{\alpha}$ has no sensitivity to the sky location being considered $\left(F_{\alpha}^{+}=0=\right.$ $\left.F_{\alpha}^{\times}\right)$or if it is much noisier than the other detectors $\left(S_{\alpha} \rightarrow\right.$ $\infty)$. In these cases, the expression (A9) at that frequency and sky position reduces to that for the network that does not include detector $\alpha$.

See Rakhmanov [32] for a discussion of singularities in waveform reconstruction due to rank-deficiency of $\boldsymbol{F}_{\mathrm{w}}$ and the use of Tikhonov regularization to avoid such problems. Rakhmanov's expressions correspond to ours with the replacement $F_{\alpha}^{+, \times} \rightarrow F_{\mathrm{w} \alpha}^{+, \times}$, i.e., replacing the antenna responses by the noise-weighted antenna responses.

\section{APPENDIX B: NULL-STREAM PROJECTION OPERATOR}

Using the results of the previous appendix, it is trivial to write down an explicit expression for the projection operator that acts on the network data vector to produce null streams. This projection operator projects the data orthogonally to $\boldsymbol{F}_{\mathrm{w}}^{+}$and $\boldsymbol{F}_{\mathrm{w}}^{\times}$. Using the Moore-Penrose inverse $\boldsymbol{F}_{\text {MP }}^{-1}$ (A5) of the antenna response matrix $\boldsymbol{F}$, we see immediately that

$$
\boldsymbol{P}^{\mathrm{NS}}=\boldsymbol{I}-\boldsymbol{F}_{\mathrm{w}} \boldsymbol{F}_{\mathrm{MP}}^{-1}
$$

projects the data orthogonally to $\boldsymbol{F}_{\mathrm{w}}^{+}$and $\boldsymbol{F}_{\mathrm{w}}^{\times}$. If $\boldsymbol{F}_{\mathrm{w}}^{+} \propto \boldsymbol{F}_{\mathrm{w}}^{\times}$ (e.g., for co-aligned detectors) then the projection operator simplifies to

$$
P^{\mathrm{NS}}=\boldsymbol{I}-\frac{\boldsymbol{F}_{\mathrm{w}}^{+} \boldsymbol{F}_{\mathrm{w}}^{+^{T}}}{\boldsymbol{F}_{++}} .
$$

Note that these projection operators map the $D$-dimensional data vector into another $D$-dimensional vector rather than a $(D-r)$-dimensional vector; however, they only span a $(D-r)$-dimensional space. The explicit construction of $\boldsymbol{A}$ (14) eliminates the extra dimensions and directly yields $D-r$ null streams.
[1] The GEO-600 project home page is at http:// www.geo600.uni-hannover.de/.

[2] The LIGO project home page is at http://www.ligo. caltech.edu/.

[3] The TAMA project home page is at http://tamago.mtk. nao.ac.jp/.

[4] The Virgo project home page is at http://www. virgo.infn.it/.

[5] E. E. Flanagan and S. A. Hughes, Phys. Rev. D 57, 4535 (1998).

[6] E. E. Flanagan and S. A. Hughes, Phys. Rev. D 57, 4566 (1998).

[7] F. Pretorius, Phys. Rev. Lett. 95, 121101 (2005).

[8] J. G. Baker, J. Centrella, D.-I. Choi, M. Koppitz, and J. van Meter, Phys. Rev. Lett. 96, 111102 (2006).

[9] M. Campanelli, C. O. Lousto, P. Marronetti, and Y. Zlochower, Phys. Rev. Lett. 96, 111101 (2006).

[10] P. Diener, F. Herrmann, D. Pollney, E. Schnetter, E. Seidel, R. Takahashi, J. Thornburg, and J. Ventrella, Phys. Rev. Lett. 96, 121101 (2006).

[11] F. Herrmann, D. Shoemaker, and P. Laguna, gr-qc/ 0601026.

[12] F. Loffler, L. Rezzolla, and M. Ansorg, gr-qc/0606104.

[13] M. Shibata and K. Taniguchi, Phys. Rev. D 73, 064027 (2006).

[14] T. Zwerger and E. Müller, Astron. Astrophys. 320, 209 (1997).
[15] H. Dimmelmeier, J. Font, and E. Muller, Astron. Astrophys. 393, 523 (2002).

[16] C. Ott, A. Burrows, E. Livne, and R. Walder, Astrophys. J. 600, 834 (2004).

[17] M. Shibata and Y. I. Sekiguchi, Phys. Rev. D 69, 084024 (2004).

[18] C. D. Ott, A. Burrows, L. Dessart, and E. Livne, Phys. Rev. Lett. 96, 201102 (2006).

[19] P. Mészáros, Annu. Rev. Astron. Astrophys. 40, 137 (2002).

[20] C. Cutler and K.S. Thorne, gr-qc/0204090.

[21] B. Allen, Phys. Rev. D 71, 062001 (2005).

[22] B. Abbott et al. (LIGO Scientific Collaboration), Phys. Rev. D 72, 082001 (2005).

[23] B. Abbott et al. (LIGO Scientific Collaboration), Phys. Rev. D 72, 062001 (2005).

[24] B. Abbott et al. (LIGO Scientific Collaboration), Classical Quantum Gravity 23, S29 (2006).

[25] L. Cadonati, Classical Quantum Gravity 21, S1695 (2004).

[26] B. Abbott et al. (LIGO Scientific Collaboration), Phys. Rev. D 72, 122004 (2005).

[27] Y. Gürsel and M. Tinto, Phys. Rev. D 40, 3884 (1989).

[28] W. G. Anderson, P. R. Brady, J. D. E. Creighton, and E. E. Flanagan, Phys. Rev. D 63, 042003 (2001).

[29] S. Klimenko, S. Mohanty, M. Rakhmanov, and G. Mitselmakher, Phys. Rev. D 72, 122002 (2005).

[30] S. Klimenko, S. Mohanty, M. Rakhmanov, and G. 
Mitselmakher, J. Phys.: Conf. Ser. 32, 12 (2006).

[31] S. Mohanty, M. Rakhmanov, S. Klimenko, and G. Mitselmakher, Classical Quantum Gravity 23, 4799 (2006).

[32] M. Rakhmanov, gr-qc/0604005.

[33] J. Sylvestre, Phys. Rev. D 68, 102005 (2003).

[34] N. Arnaud, M. Barsuglia, M.-A. Bizouard, V. Brisson, F. Cavalier, M. Davier, P. Hello, S. Kreckelbergh, and E. K. Porter, Phys. Rev. D 68, 102001 (2003).

[35] M. Tinto, J. W. Armstrong, and F. B. Estabrook, Phys. Rev. D 63, 021101(R) (2000).

[36] M. Tinto and S.L. Larson, Phys. Rev. D 70, 062002 (2004).

[37] L. Wen and B. Schutz, Classical Quantum Gravity 22, S1321 (2005).

[38] Any gravitational wave at the Earth, produced by a source at astronomical distances, can be regarded as a plane wave.

[39] The null space of $\boldsymbol{F}_{\mathrm{w}}{ }^{T}$ is the set of all vectors $\boldsymbol{x}$ such that $\boldsymbol{F}_{\mathrm{w}}{ }^{T} \boldsymbol{x}=0$.

[40] G. Strang, Introduction to Linear Algebra (WellesleyCambridge Press, Wellesley, Massachusetts, 1998), 3rd ed..

[41] S. Chatterji, L. Blackburn, G. Martin, and E. Katsavounidis, Classical Quantum Gravity 21, S1809
(2004).

[42] S. Chatterji, PhD dissertation, MIT, 2004.

[43] M. Tinto, in Proceedings of the International Conference on Gravitational Waves: Source and Detectors (World Scientific, Singapore, 1996).

[44] A. Dietz, J. Garofoli, G. Gonzalez, M. Landry, B. O'Reilly, and M. Sung, LIGO Document: Report No. LIGO-T050262-D, 2005.

[45] The XPIPELINE CVS repository is at http://www.lscgroup.phys.uwm.edu/cgi-bin/cvs/viewcvs.cgi/matapps/ src/searches/burst/coherent-network/?cvsroot=lscsoft.

[46] http://www.ligo.caltech.edu/docs/T/T970130-F.pdf.

[47] W. Althouse et al., Rev. Sci. Instrum. 72, 3086 (2001).

[48] F. Beauville et al., J. Phys.: Conf. Ser. 32, 212 (2006).

[49] XPIPELINE can also process multiple frequency bands; we use a single band in this demonstration for simplicity.

[50] http://www.ligo.caltech.edu/lazz/distribution/LSC_Data/.

[51] J. Baker, M. Campanelli, C. O. Lousto, and R. Takahashi, Phys. Rev. D 65, 124012 (2002).

[52] A. Lazzarini, S. Bose, P. Fritschel, M. McHugh, T. Regimbau, K. Reilly, J.D. Romano, J. T. Whelan, S. Whitcomb, and B.F. Whiting, Phys. Rev. D 70, 062001 (2004).

[53] P. Ajith, M. Hewitson, and I. S. Heng, gr-qc/0604004. 